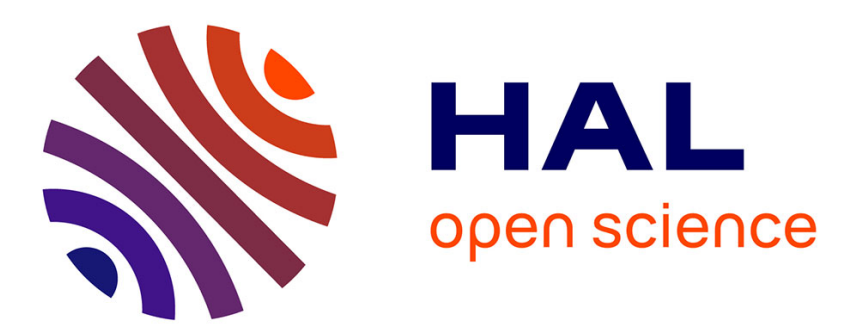

\title{
Millisecond dynamics of deformation bands during discontinuous creep in an AlMg polycrystal
}

\author{
A A Shibkov, M A Lebyodkin, T A Lebedkina, M F Gasanov, A E Zolotov, A
} A Denisov

\section{- To cite this version:}

A A Shibkov, M A Lebyodkin, T A Lebedkina, M F Gasanov, A E Zolotov, et al.. Millisecond dynamics of deformation bands during discontinuous creep in an AlMg polycrystal. Physical Review E , 2020, 102, pp.043003. 10.1103/physreve.102.043003 . hal-02994009

\section{HAL Id: hal-02994009 \\ https://cnrs.hal.science/hal-02994009}

Submitted on 7 Nov 2020

HAL is a multi-disciplinary open access archive for the deposit and dissemination of scientific research documents, whether they are published or not. The documents may come from teaching and research institutions in France or abroad, or from public or private research centers.
L'archive ouverte pluridisciplinaire HAL, est destinée au dépôt et à la diffusion de documents scientifiques de niveau recherche, publiés ou non, émanant des établissements d'enseignement et de recherche français ou étrangers, des laboratoires publics ou privés. 


\title{
Millisecond dynamics of deformation bands during discontinuous creep in an AlMg polycrystal
}

\author{
A.A. Shibkov, ${ }^{1 *}$ M.A. Lebyodkin, ${ }^{2}$ T.A. Lebedkina, ${ }^{2,3}$ \\ M.F. Gasanov, ${ }^{1}$ A.E. Zolotov, ${ }^{1}$ A.A. Denisov ${ }^{1}$ \\ ${ }^{1}$ Physics department, Tambov State University, Internationalnaya str., 33, 392000 Tambov, Russia \\ ${ }^{2}$ Laboratoire d'Etude des Microstructures et de Mécanique des Matériaux (LEM3), CNRS, \\ Université de Lorraine, Arts \& Métiers ParisTech, 7 rue Félix Savart, 57070 Metz, France \\ ${ }^{3}$ Institut de recherche technologique-Matériaux, Métallurgie et Procédés (IRT M2P), 4 rue \\ Augustin Fresnel, 57070 Metz, France
}

\begin{abstract}
Formation of bands of macroscopic strain localization during staircase creep in an $\mathrm{AlMg}$ polycrystal is studied by the acoustic emission (AE) technique and high-speed video-recording with an image acquisition rate up to 50000 frames per second. The simultaneous measurements by two methods allow to distinguish different types of embryo deformation bands and concomitant AE signals and establish correlations between the band evolution and the acoustic response. It is found that the fastest stages of band formation, associated with its emergence to the surface and subsequent accelerated expansion, generate complex AE bursts in the frequency band $\sim 0.05-1 \mathrm{MHz}$. The correlations hidden in the complex structure of an individual acoustic burst are investigated by methods of statistical and fractal analysis. On the other hand, relationships between average parameters of various physical responses to discontinuous creep are assessed. Particularly, a close correspondence is found between the envelope of the acoustic burst and the rate of stress change during formation of a single deformation band. Evolution of dynamical behavior of embryo bands with increasing creep stress is discussed. Notably, a qualitative change in the $\mathrm{AE}$ waveform observed on approaching the ultimate stress is considered from the viewpoint of anticipation of the oncoming fracture.
\end{abstract}

* Corresponding author. E-mail address: shibkov@tsu.tmb.ru

\section{INTRODUCTION}

Self-organization of dislocations gives rise to nonlinear effects in the macrokinetics of plastic deformation of crystals, which are attracting increasing interest since the 1980s. The most striking example of such effects is presented by intermittent deformation of alloys, which manifests itself in two different situations: (1) Stepwise strain-time curves, $\varepsilon(t)$, are observed when the sample is loaded in a soft deformation machine with a given rate of growth of applied stress, $\dot{\sigma}_{0}=$ const. Such instability of uniform plastic flow is known as the Savart-Masson effect [1]; A special case corresponding to the condition $\dot{\sigma}_{0}=0$, i.e., $\sigma_{0}=$ const, is distinguished as discontinuous (staircase) creep [2]. (2) The Portevin-Le Chatelier (PLC) effect is manifested by serrated stress-time curves, $\sigma(t)$, observed during deformation at an imposed strain rate, $\dot{\varepsilon}_{0}=$ const, in a rigid testing machine [3]. The former case corresponds to the direct problem of dynamics, addressing the study of motion (here, unstable deformation) under the action of given 
forces. The latter corresponds to the inverse problem, i.e., the force response of the mechanical machine-sample system is studied for a given evolution of strain.

According to the generally accepted point of view, the plastic instabilities are caused by the phenomenon of dynamic strain ageing (DSA), i.e., by the dynamic interaction of mobile dislocations with diffusing impurity atoms [4]. The underlying physics is that there occurs a plastic strain-rate interval where the pinning force on the dislocation temporarily immobilized on obstacles and waiting for thermal activation decreases with the increase in the strain rate because a smaller number of solute atoms reach the dislocation during the respectively diminished waiting time. In the mathematical sense, this mechanism gives rise to a negative sensitivity of stress to the strain rate in some interval. The resulting nonlinear $N$-shaped material characteristic reveals the collective dynamics of dislocations as a pinning-unpinning problem and relates it to behavior of diverse nonlinear systems controlled by stick-slip phenomena and characterized by velocity-weakening friction $[5,6]$. In particular, creep deformation is a basic element of tectonics that includes periods of slow continuous creep alternating with abrupt earthquakes [7]. Consequently, intermittent deformation of alloys is often considered from a larger aspect than that of pure plasticity problems, namely, as a laboratory model for experimental investigation of collective phenomena in extended systems [8-10].

In both cases described above, intermittent deformation is associated with drastic spatial localization of plastic strain in macroscopic bands [11, 12]. It was understood quite early, e.g., using capacitance strain transducers, that the development of a deformation band may proceed very fast, during times shorter than a millisecond [13]. However, as the techniques of visualization of strain localizations were operating in a range of about 10 to 50 frames per second (fps), the question of their nucleation and formation has been little explored. Investigations of intermittent plasticity were mainly aimed at determination of average parameters of complete deformation bands and correlations between them, the latter concerning complex spatial patterns of deformation bands and concomitant temporal patterns of serrations on the scale of a deformation curve. Most of studies were devoted to the PLC effect which provides large amounts of data because, as the total plastic strain rate caused by a deformation band exceeds $\dot{\varepsilon}_{0}$, it results in elastic unloading that terminates the further development of the instability and allows for multiple repetitions of instability events (see reviews [8-10, 14] and references therein). It was found that when $\dot{\varepsilon}_{0}$ is increased, the behavior in conventional tensile tests changes from static deformation bands occurring at random to relay-race series of bands, each nucleated close to the boundary of the previous one, and finally to quasi-continuous propagation of deformation bands along the entire specimen [11, 14]. Investigations of the last three decades [8-10, 15-17] identified these changes with transitions between well-known dynamical modes including self-organized criticality [18], deterministic chaos [19] and synchronization [20]. This richness of behaviors attests the plastic instability as a unique object of research, allowing for testing distinct dynamical regimes by varying loading conditions. Moreover, application of high-resolution techniques based on the recording of acoustic emission (AE) [15-17] or local strain fields [21] made it possible to attack the question of relationship between dynamic strain fluctuations on the mesoscopic and macroscopic scales.

Collective effects were much less explored for the constant $\dot{\sigma}_{0}$ conditions. This loading mode leads to qualitatively different deformation patterns because the stress relaxation is impeded and the instability is much more drastic. Tensile specimens sustain only few instabilities before fracture, each giving rise to a large strain jump, sometimes up to $10 \%$. This catastrophic process was believed to be related to irregular quasi-continuous propagation of deformation bands [22]. Recently, implementation of video-recording with a 500-fps acquisition rate allowed to show that a single instability event (a strain jump lasting about one second) during creep of an AlMg alloy is produced by cascade nucleation of several tens of deformation bands [12]. The occurrence of each band is accompanied by a stress serration due to partial elastic unloading. Moreover, since the plastic strain rate varies during the development of the 
jump, transitions between regimes of cascade nucleation and continuous propagation of deformation bands were detected. Thus, although such a pattern refers to one strain jump during creep, it presents analogies with patterns characterizing series of PLC bands. These observations, waiting for a rigorous mathematical interpretation, bear evidence to an important role of longrange elastic stresses which, on the one hand, give rise to diverse spatiotemporal patterns when the conditions for stress relaxation are varied, and, on the one hand, may be responsible for similitudes between behaviors occurring in different conditions or on different physical scales.

This consideration strengthens the interest to the mechanisms governing the short-time scale pertaining to the formation of individual bands in various conditions. The last decade exhibited an important progress in this sense due to improvements of the time resolution of visualization of the surface of a deforming sample. Application of various optical techniques with an acquisition rate of 1000 to $5000 \mathrm{fps}$ made it possible to reveal nucleation of an embryo of a PLC band on one side of the specimen and its growth in the direction of the maximum tangential stresses, about $60^{\circ}$ to the tensile axis [23-25]. To complete the picture, it should be added that quasi-simultaneous strain localization across the specimen was also observed $[25,26]$. In this case, the further evolution of the deformation band consists of a progressive growth of the local strain rate profile, while its width remains unchanged (cf. [27, 28]). Nucleation and growth of an embryo band was also found for creep. Moreover, video-recording with a speed of 25000 fps allowed to characterize an elliptic shape of the embryo band contour, jumps in the velocity of its tip, and the instant of its arrival to the opposite side of a flat sample, i.e., the end of formation of a complete deformation band [29, 30].

As can be seen, the increase in the time resolution by three orders of magnitude (from 10 to $25000 \mathrm{fps}$ ), and also a several times increase in spatial resolution (from about 100 to 8 $\mu \mathrm{m} / \mathrm{pixel}$ ), allowed for revealing significant features of geometry and dynamics of individual deformation bands at the earliest stage of their evolution. The time scales characterizing an embryo band evolution, $\sim 0.1-1 \mathrm{~ms}$, are inferior to the front duration of the subsequent unloading jump ( 1-3 ms). Both characteristic times are by orders of magnitude shorter than the time intervals between jumps, even in the conditions of stress control. This sharp distinction between time scales separates the processes responsible for the occurrence and growth of an embryo band into a particular problem that may not be directly related to dynamic behavior on larger scales corresponding to lateral motions and correlations between complete bands. This suggestion is corroborated by recent studies with the aid of the $\mathrm{AE}$ technique, which revealed a complex structure of acoustic signals accompanying individual PLC bands [31]. Thus, recent progress in the investigations of jerky flow emphasizes the first millisecond of the evolution of a deformation band as a self-contained object of study. An in situ investigation of "millisecond dynamics" of formation of individual deformation bands requires the use of methods with a high temporal and spatial resolution, such as video-recording of the surface of a deforming sample at a rate of at least $5000 \mathrm{fps}$, supported by appropriate computer-based image processing and synchronized with other high-frequency methods, e.g., the AE technique providing complementary information inaccessible to optical methods.

In the previous works by the authors [29, 30], the dynamics of deformation bands was studied under conditions of discontinuous creep of an AlMg6 alloy with the aid of the AE method in the low-frequency range of $\sim 10 \mathrm{~Hz}-10 \mathrm{kHz}$ and synchronous video-recording of the specimen surface with a time resolution of 40-50 $\mu$ s. It allowed to establish that the main AE occurs when the growing embryo band reaches the opposite side of the specimen and to correlate the jump in the stress drop rate with the first AE peak with a rise time of $\sim 1 \mathrm{~ms}$. In particular, this correspondence provided experimental evidence for the mechanism of AE generation by the dislocations emerging on the sample surface. The objective of the present work was two-fold. First, it was aimed at assessing finer-scale dynamics and morphology of deformation bands during discontinuous creep, revealed by recording discrete $\mathrm{AE}$ in an ultrasonic range, from $\sim 25$ $\mathrm{kHz}$ to $1 \mathrm{MHz}$, and video-recording of the specimen surface at a rate up to $50000 \mathrm{fps}$. Second, this approach uncovered a wealth of features that had not been accessible in earlier works and 
allowed for an analysis of correlations of deformation processes on the time scale of individual deformation bands (cf. [31]). First attempts of such analysis were undertaken using statistical and fractal formalisms.

The paper is organized as follows. Section II describes the research methodology and experimental techniques. Section III presents experimental results and discussion on the following issues: specific types of embryo deformation bands, their acoustic signatures, information carried by $\mathrm{AE}$ signals with regard to the dynamics of deformation processes. The nonlinear growth of embryo bands and their action as a source of AE is discussed in view of the results obtained. The concluding section IV provides a summary of the main findings.

\section{EXPERIMENTAL PROCEDURE AND DATA PROCESSING}

Details of the experiment and methodological aspects were described elsewhere $[12,30]$ and will be briefly outlined here. Tensile specimens with a dog-bone shape and a gauge size of $6 \times 3 \times 0.2$ $\mathrm{mm}^{3}$ were cut along the rolling direction from a cold-rolled sheet of a polycrystalline aluminummagnesium AlMg6 (AA5059) alloy with the chemical composition Al-6.15\%Mg-0.65\%Mn$0.25 \% \mathrm{Si}-0.21 \% \mathrm{Fe}$ (wt.\%). After annealing for one hour at $450{ }^{\circ} \mathrm{C}$ and quenching in water, the average grain size was about 10-12 $\mu \mathrm{m}$. The specimens were deformed in air at room temperature in a constant-load lever-arm creep test machine. The tests consisted of two stages. First, the sample was loaded at $\dot{\sigma}_{0}=0.1 \mathrm{MPa} / \mathrm{s}$ to a fixed stress in a range from 200 to $320 \mathrm{MPa}$ considerably exceeding the conventional yield point for an AlMg6 alloy, $\sigma_{0} \approx 160 \mathrm{MPa}$. It was then kept at this stress corresponding to discontinuous creep conditions.

Various parameters were followed to characterize the dynamics of discontinuous creep. First, as mentioned in the Introduction, strain bursts caused by plastic instabilities lead to partial elastic unloading due to inertia of the deformation machine. The force response to the development of deformation bands was measured using a Zemic H3-C3-100 kg-3V load sensor. AE signals were recorded using a Zetlab BC-601 sensor with an almost flat response in the frequency range of $100-800 \mathrm{kHz}$ and a smooth decrease at lower frequencies, $<100 \mathrm{kHz}$. The sensor was attached to the specimen head close to the gauge part, using a silicon grease to assure a good acoustic contact. The voltage from the sensor was amplified by $40 \mathrm{~dB}$ with the aid of a AEP5 preamplifier (Vallen-Systeme) and recorded continuously without a threshold at a $5 \mathrm{MHz}$ sampling rate and 16-bit amplitude resolution. The low-frequency part of the AE spectrum, studied earlier in $[29,30]$, was suppressed by high-pass filters set at $25 \mathrm{kHz}, 50 \mathrm{kHz}$, or $100 \mathrm{kHz}$ depending on the research tasks.

A high-speed digital device camera FASTCAM Mini UX50/100 (Photron) was applied to monitor the surface of the deforming sample synchronously with the force and strain measurements. The image acquisition rate was varied from 5000 to $50000 \mathrm{fps}$ in order to survey the early stages of formation of deformation bands. The sample surface was illuminated by a white light source at an angle of $30-40^{\circ}$. The optical technique applied in this work is similar to the shadow method [11] modified in two aspects: (i) More than a three-orders-of-magnitude increase in the video-recording speed and (ii) Computer-assisted processing based on subtraction of sequential digital images.

\section{EXPERIMENTAL RESULTS AND DISCUSSION}

\section{A. Types of embryo deformation bands}

The analysis of video records of the initial stage of formation of more than one hundred individual deformation bands, lasting about a millisecond in the conditions of discontinuous creep, allowed to classify the embryo bands into at least four main types according to their morphology and dynamics. The first type refers to primary "mother" bands which nucleate and grow in a material without prior deformation bands (Fig. 1a). The second type includes 
secondary bands that arise near the boundary of a previous deformation band (including the primary one) and grow along it (Fig. 1b). The conjugated embryo band, which grows at an angle of about $60^{\circ}$ to the previous bands, belongs to the third type (Fig. 1c). Finally, the fourth type includes arched bands growing in the region of the specimen shoulders (Fig. 1d).

Embryo bands of different type are characterized by different dynamics. The dynamics and geometry of the first two types are identical, as described in detail in [12, 29, 30]. Up to about half the cross section of a flat sample, the embryo band tip grows at an approximately constant velocity at an angle of $58-63^{\circ}$ to the tensile axis. Then starts the stage of accelerated tip growth. It obeys an exponential law, $v_{t} \sim \exp (t / \tau)$, with the time constant $\tau \sim 0.1-0.2 \mathrm{~ms}$. The velocity reaches maximum when the band exits to the opposite side surface of the flat sample. Its value is often limited by the video-recording speed: $\sim 3-5 \mathrm{~m} / \mathrm{s}$ at the sampling rate of $500 \mathrm{fps}, \sim 10-15$ $\mathrm{m} / \mathrm{s}$ at $25000 \mathrm{fps}$ and $\sim 30-40 \mathrm{~m} / \mathrm{s}$ at $50000 \mathrm{fps}$.

It is known that the deformation band represents a mechanical charge, i.e., contains an excess of dislocations of one mechanical sign [32]. The corresponding bending moment is compensated by local deformations with an excess of the opposite mechanical charge, notably by conjugate deformation bands. Thus, conjugate embryo bands belonging to the third type inevitably arise during deformation. They are characterized by a slower growth kinetics exhibiting variations in the tip velocity in the regions of intersection with the previously formed bands. The slower kinetics is not surprising because in this case, the band moves through material with a macroscopically inhomogeneous field of dislocation pinning forces created by its predecessors. The jump in the growth rate upon reaching the opposite lateral surface is less pronounced; the maximum tip velocity does not usually exceed $\sim 5 \mathrm{~m} / \mathrm{s}$. It should be noted that the interaction of conjugate bands is important in the formation of the final neck and fracture of the sample [33-35]. It can be assumed that when the conjugate bands intersect, numerous microcracks are formed according to the Cottrell mechanism [36], merging into the main crack at high stresses.

Arcuate embryo bands belonging to the fourth type grow in the region of specimen heads where the stress state essentially differs from that in the gauge part and the stress level is reduced due to an increasing cross-section. The growth rate of the tip of such bands is the lowest, not exceeding several meters per second. The growth duration is accordingly the longest, up to several milliseconds.

\section{B. Acoustic signals generated during formation of deformation bands}

Despite individual features of acoustic bursts generated during formation of deformation bands, including the stage of the embryo band growth, they can be divided into two main groups according to their amplitude-frequency parameters. The first group includes the most common acoustic signals represented by broadband bursts in the frequency range $\sim 25 \mathrm{kHz}-1 \mathrm{MHz}$ and with a duration of $\sim 1-10 \mathrm{~ms}$ (Fig. 2a). Such events are generated by the third and fourth type bands, as well as by the first and second type bands at stresses up to approximately 270-290 $\mathrm{MPa}$. At higher stresses until failure, the bands of the first and second type generate short signals with durations up to $\sim 1 \mathrm{~ms}$ forming the second group of AE responses. They are represented by series of high-amplitude microsecond pulses separated by intervals of low-frequency damped oscillations (Fig. 2b). These intervals usually last for $\sim 50-200 \mu \mathrm{s}$. The first microsecond pulse of the series indicates the onset of the stage of accelerated growth of the embryo band tip. One of the subsequent high-amplitude microsecond pulses marks the end of this stage corresponding to the band exit to the opposite sample's surface, i.e., to formation of a complete deformation band. Then, the series of microsecond pulses usually continues as long as $\sim 200-500 \mu \mathrm{s}$.

It is noteworthy that a similar separation of two groups of acoustic signals accompanying intermittent deformation, according to their amplitude-frequency characteristics, is also known from experimental studies of AE signals generated during stress jumps caused by the PLC effect 
in AlMg alloys [15, 37-39]. Thus, such signals are typical not only for "hard" ( $\dot{\varepsilon}_{0}=$ const), but also for "soft" loading regime under discontinuous creep conditions ( $\sigma_{0}=$ const). In view of the general character of these temporal patterns, next sections undertake quantitative analyses of both kinds of acoustic signals - broadband bursts and short series of microsecond AE pulses and their correlations with the dynamics of embryo deformation bands.

C. Dynamics of deformation band formation reflected in broadband AE bursts

\section{Correlation of the AE burst envelope with the deformation band growth and the force response}

As stated in the Introduction, the strain jump with a typical amplitude of $\sim 1-5 \%$ on the creep curve represents a complex process involving the development of multiple bands of localized plastic strain (see Fig. 4 and 5 in Ref. [12]). In this Section, the very first band triggering the entire process will be considered in detail. It occurs after some incubation time depending on the creep stress reached upon the initial preloading, from 270 to $290 \mathrm{MPa}$ in the following examples. The video-recording of the sample surface shows that the band spontaneously arises and grows at a random position along the gauge part or near one of the specimen shoulders. The latter case is shown in Fig. 3 which displays a fragment of recording of the process of nucleation and growth of an incomplete arcuate deformation band.

Figure 4 presents results of the image processing in the form of kinetic curves for the growth of the band length $L_{b}$, the tip velocity $v_{t}$, the band area $S_{b}$, i.e., the area enclosed between the band boundaries, and its growth rate $\dot{S}_{b}$; The corresponding acoustic signal $\varphi_{A E}(t)$ is displayed in the bottom chart (Fig. 4e). The time dependences of geometric parameters of the incomplete band demonstrate typical nonlinear dynamics with a sharp acceleration at the final growth stage. It should be recalled that due to a lower stress level in the region of the specimen head, the growth rate of incomplete arcuate bands is less than the band growth rate in the central region of the gauge part, where the band grows in the direction of maximum tangential stresses $[23-26,30]$. It can be seen that the instant of the band exit to the opposite side surface corresponds to peaks in $\varphi_{A E}(t), v_{t}$ and $\dot{S}$. Unlike the low-frequency signal of discrete AE in the $10 \mathrm{~Hz}-10 \mathrm{kHz}$ band (see [29, 30]), the acoustic burst shown in Fig. 4 has a more complex structure in a wide frequency band (Fig. 5), from the low frequency of $50 \mathrm{kHz}$, determined by the high-pass filter, and up to $\sim 1 \mathrm{MHz}$, this bound mainly due to the upper frequency response of the AE sensor.

Thus, the formation of a deformation band is accompanied by a broadband acoustic burst, the power spectrum $P(f)$ of which contains virtually all frequencies of the studied range, with the main peak near $100 \mathrm{kHz}$ (Fig. 5) and the median frequency $f_{m}$ of $300 \mathrm{kHz}$, as determined from the

condition $\int_{0}^{f_{m}} P(f) d f=\int_{f_{m}}^{\infty} P(f) d f \quad[40]$. The envelope of the AE signal is bell-shaped with a maximum corresponding to the end of formation of the complete deformation band (Figs. 4 and 6).

It follows from Fig. 4 that the main contribution to the acoustic burst comes from the stage of accelerated growth of the incomplete band before it reaches the opposite surface of the sample, i.e., before the instant corresponding to the formation of a complete deformation band. This stage approximately covers the second half of the period of growth of the band length (frames 970-988 in Fig. 3). The preceding stage with the band tip velocity $v_{t}<0.5 \mathrm{~m} / \mathrm{s}$, including the moment of the band initiation, renders a relatively small AE signal that does not exceed the level of the continuous AE component. The stage of attenuation of the acoustic burst after formation of the complete band (frame 988 in Fig. 3) approximately coincides with the stage of attenuation of the growth rate of the band area, $\dot{S}_{b}(t)$, with a duration of $\sim 1 \mathrm{~ms}$. This stage of the acoustic burst is presumably governed by the collective dynamics of dislocations 
inside the band, but perhaps also by the reflections of acoustic waves from the sample surfaces. The latter, however, are only possible at frequencies $f_{\max }>1 \mathrm{MHz}$, when the acoustic wavelength $\lambda$ is comparable to or less than the sample size: $\lambda=c / f_{\max } \approx 5 \mathrm{~mm}$, where the speed of sound $c \approx 5 \cdot 10^{3} \mathrm{~m} / \mathrm{s}$. As can be seen from Fig. 5, this spectral region ("high-frequency tail") constitutes only a small part $(<10 \%)$ of the power spectrum. Therefore, the possible effects of the reflection of acoustic waves at frequencies of $\sim 1 \mathrm{MHz}$ cannot explain the complex broadband structure of the AE burst generated during the deformation band formation.

Since the typical duration of the broadband AE burst is of the order of $\sim 3-10 \mathrm{~ms}$, i.e., commensurate with the duration of the stress drop front, it is of interest to study the correlation of the averaged envelope of the AE burst with the force response or, more precisely, with the shape of the function $\psi(t)=|d \sigma(t) / d t|$. Figure 6 displays the force and acoustic responses to the formation of an individual deformation band, as represented by the unloading jump $\sigma(t)$ and the acoustic burst $\varphi_{A E}(t)$, for comparison with the shape of the function $\psi(t)$. The averaged envelope of the acoustic burst was calculated according to equation:

$$
\xi(t)=<\left|\varphi_{A E}(t)\right|>=\Delta t^{-1} \int_{t}^{t+\Delta t}\left|\varphi_{A E}\left(t^{\prime}\right)\right| d t^{\prime},
$$

where $\Delta t$ and $\left|\varphi_{A E}(t)\right|$ designate the averaging time and the absolute value of the AE signal, respectively. Since the load was recorded at a rate of $2 \mathrm{kHz}, \Delta t$ was chosen equal to $0.5 \mathrm{~ms}$. Bell-shaped time dependences $\xi(t)$ and $\psi(\xi)$ averaged over $\Delta t$ are presented in Fig. 6 (curves $\mathrm{d}$ and e, respectively). The correlation coefficient of these dependences, calculated using the MathCad software package, $k \approx 0.9316$.

Another sign of correlation of these functions is an approximately linear dependence between their amplitudes $\Delta \xi_{m}$ and $\Delta \psi_{m}$ (Fig. 7). Thus, the averaged envelope of the absolute value of the high-frequency acoustic signal carries information about the rate of change of the stress response $|\dot{\sigma}|$. This result qualitatively agrees with the conclusions of Ref. [30], which reported on the correlations of the function $\psi(t)$ with the amplitude of the low-frequency AE signals caused by the formation of individual deformation bands and measured in the band of 10 $\mathrm{Hz}-10 \mathrm{kHz}$.

Figure 8 bears evidence that the acoustic burst accompanying the formation of a single deformation band has a complex, apparently fractal structure consisting of a large number of short, $\sim 1-10 \mu \mathrm{s}$, discrete pulses of various amplitudes. Taking into account the general hypothesis of an avalanche nature of collective dislocation movements [16, 17, 41-47], it is natural to assume that these $\mathrm{AE}$ pulses are associated with dislocation avalanches of various power participating in the evolution of a complex spatiotemporal pattern of the macroscopic strain localization. Since avalanche behavior is characterized by scale-invariant statistical properties, a deeper insight into this complexity may be provided by a study of the temporal structure of acoustic bursts using methods of statistical, correlation, and fractal analyses.

\section{Statistical and fractal analysis of the structure of a broadband AE burst}

To perform a statistical analysis, first, a lower threshold of the AE signal amplitude was set at the level of twice the RMS value of the signal recorded a few seconds before the onset of the primary deformation band (approximately in the middle of the plateau between steps on the creep curve). Then, the total number of AE pulses above this threshold was counted (in the example of Fig. 8, $N=2643$ ). The probability distribution function (PDF), $D(s)=N^{-1} d N / d s$,

was calculated for the normalized acoustic pulse energy, $s=A^{2} / \overline{A^{2}}$, where $A=\varphi_{m}$ stands for the amplitude of an individual pulse in the AE burst, its square $A^{2}$ characterizes the pulse energy 
$E, \overline{A^{2}}$ is the respective average value over the burst, and $d N$ gives the number of AE pulses with amplitudes in a narrow interval $[s-\delta s / 2, s+\delta s / 2]$.

The PDF of the squared amplitudes presented in Fig. 9 has a hyperbolic shape, radically different from a normal distribution characterizing random processes. The $D(q)$ dependence is approximately linear in log-log coordinates which means that the amplitude distribution obeys a power law

$$
D(s) \sim s^{-\beta}
$$

The estimation of the slope of the log-log dependence renders the value of the exponent $\beta=2.2 \pm 0.1$. The power-law distribution of squared amplitudes of AE pulses indicates selfsimilarity of the acoustic signal, i.e., the absence of a characteristic scale of events, relevant of fractal time series [48, 49].

This result is consistent with the data of numerical simulation of self-organizing dislocation avalanches at a steady-state creep [41], as well as with experimentally found statistics of AE signals during creep of ice single crystals [42, 43] and under conditions of the PLC effect in metals $[16,44]$. It is important to specify that the cited works dealt with a much coarser scale corresponding to series of AE bursts, so that each burst provided one data point while its intrinsic structure was ignored. Such similitude corroborates the conjecture of an inherently scaleinvariant nature of dislocation dynamics in various conditions.

The numerical study on ice single crystals deforming by slip in the basal plane shows that creep results in the formation of dislocation dipoles and walls. These defects determine the pinning force field and lead to formation of dislocation avalanches of different strength, so that the amplitude distribution of AE signals demonstrates a power law [41]. It is natural to suggest that the quantitative law depends on the material microstructure. Indeed, experimental data for $\beta$ vary from 1.4 to 2 for various materials, displaying lower values for crystals with hexagonal lattices (ice, $\mathrm{Cu}, \mathrm{Zn}, \mathrm{Cd}$ ) and higher values for cubic crystals (the maximum value of 2 was found for Al) [45]. Experimental studies of discrete AE under conditions of the PLC effect in polycrystalline binary Al-Mg alloys with magnesium content of $3-6 \%$ give $\beta$ in a range from 2 to $3[17,44]$, and even higher for Al-Mg alloys with precipitates [16]. As suggested in [17], the larger values of $\beta$ may be due to the multiple slip, the presence of precipitates, grain boundaries, forest dislocations, and impurity atoms, which are effective obstacles to the dislocation motion and, therefore, increase the fraction of smaller dislocation avalanches at the expense of larger avalanches.

Taking into account the role of long-range stresses in the correlation of collective deformation processes, it is worth recalling earthquakes as a classical example of selforganization in natural mechanical systems. Their magnitudes are measured in terms of the energy released by tectonic plates sliding, and the corresponding statistical distributions obey power laws with an exponent usually ranging from 1.8 to 2.2 , depending on the earthquake geography [48]. Thus, although the microscopic mechanisms of deformation are different in the laboratory and natural systems, the spontaneous formation of deformation bands at a creep stress significantly exceeding the yield strength is similar to earthquakes, regarding the threshold dynamics and statistical distributions of the energy of acoustic signals in the complex pattern of an $\mathrm{AE}$ burst. The observation of power-law amplitude distributions of AE signals - a signature of long-range correlations covering the entire system or a significant part of it - bears evidence that the dynamics of deformation band formation is essentially governed by slow accumulation of internal stresses and their subsequent spontaneous fast relaxation through self-organizing avalanches. 
Another evidence of self-similarity and hidden correlations in acoustic signals can stem from the property of fractality. To estimate the fractality of the recorded signals, rescaled range $(\mathrm{R} / \mathrm{S})$ analysis was applied to calculate the Hurst exponent $H$ according to the expression [49]:

$$
R / S \sim \tau^{H},
$$

where $R(\tau)=Y_{\max }-Y_{\min }$ is the range of the accumulated deviations of the time series representing the signal $\varphi_{A E}(t)$ from its average value over time interval $\tau$. The accumulated deviation is defined as

$$
Y(t, \tau)=\int_{0}^{t}\left[\varphi_{A E}\left(t^{\prime}\right)-\left\langle\varphi_{A E}\right\rangle_{\tau}\right] d t^{\prime}
$$

where $\left\langle\varphi_{A E}\right\rangle_{\tau}=\tau^{-1} \int_{0}^{\tau} \varphi_{A E}(t) d t$ and $S(\tau)=\left\{\tau^{-1} \int_{0}^{\tau}\left[\varphi_{A E}(t)-\left\langle\varphi_{A E}\right\rangle_{\tau}\right]^{2} d t\right\}^{1 / 2}$ give the average value and the standard deviation of $\varphi_{A E}(t)$ over $\tau$. The fractal dimension of such a signal embedded in a two-dimensional space can be defined using a relationship $D_{f}=2-H$ [49].

Figure 10 demonstrates the results of calculation of dependence (3) for the above example of acoustic signal. The curve can be approximated by a linear dependence in double logarithmic coordinates over one and half orders of magnitude of rescaled $R / S$ value. Its slope gives the estimate of the Hurst exponent, $H=0.304 \pm 0.06$. It can be concluded that the acoustic burst caused by the formation of a single deformation band has a fractal nature with dimension $D_{f} \approx$ 1.7. It may be recalled that $H=0.5$ for white noise (uncorrelated process), while the value of $H<$ 0.5 indicates antipersistence of the signal. Such an inherent correlation within the signal structure means that if its amplitude increases over a certain time interval, it should be expected to decrease in the next time interval of approximately the same duration, and vice versa, if the signal amplitude decreases within some interval, it will increase in the subsequent interval. This property of acoustic signals may reflect alternation of acoustic activity because large dislocation avalanches result in effective relaxation of internal stresses and, therefore, are followed by periods of sound attenuation. Vice versa, internal stresses are accumulated in the material during the relatively low activity of the AE signal, and their subsequent relaxation produces bursts of acoustic activity.

It is known that unbiased determination of $H$ requires very long time series [49]. In order to verify the above conclusion and besides, to assess the uniformity of fractal properties of $\mathrm{AE}$ signals, multifractal formalism was applied in terms of singularity spectra $f_{D}(\alpha)[49,50]$. Details of the mathematics of such analysis can be found in [51, 52]. Its qualitative meaning is clear from the following description of the construction of such spectra. The time interval containing the time series (in other words, the signal's "support") is covered with boxes of size $\delta t$ and a probabilistic measure is defined for each box. Here, the analyzed time series was represented by the series of peak amplitudes of AE pulses. Accordingly, the local measure $\mu_{i}(\delta t)$ of the $i$ th box was defined as a sum of all peak amplitudes within the box, normalized by the global sum over the entire interval. The singularity strength $\alpha$ of the local measure describes its scaling with regard to the box size:

$$
\mu_{i}(\delta t) \sim \delta t^{\alpha}
$$

The value of $f_{D}(\alpha)$ can be qualitatively defined as the fractal dimension of the subset of boxes corresponding to the singularity strength in the vicinity of $\alpha$ [49]. 
An example of such calculations is shown in Figure 11. The maximum $f_{D}$ value which characterizes the occupation of the support of the entire signal is close to 1. Such a nonfractal geometry means that the AE completely fills the time interval. Nevertheless, a certain span of $\alpha$ values, from about 0.9 to 1.15 , characterized by $f_{D}<1$, bears evidence to a multifractal nature of the analyzed signal. As $f_{D}$ values give fractal dimensions over one-dimensional supports of datasets corresponding to the given $\alpha$, there is no direct relationship between data of Figs. 10 and 11. However, it is important that the existence of a smooth singularity spectrum confirms the property of fractality of the AE accompanying the formation of a deformation band. In addition, it quantifies a certain heterogeneity of deformation processes, so that a simple fractal description may be insufficient.

\section{Relation of short series of AE pulses to specific features of deformation band formation}

At a creep stress of 300-320 MPa close to the ultimate strength, embryo bands of the first and second types generate short, with $\sim 1 \mathrm{~ms}$ duration, series of large-amplitude microsecond pulses separated by low-frequency damped oscillations (see Fig. 2b). In the tests presented below, the latter were suppressed by a high-pass filter set at the level of $100 \mathrm{kHz}$, which made it possible to isolate only discrete microsecond AE pulses and study their correlation with the dynamics of embryo bands. The AE signal was recorded at a sampling rate of $5 \mathrm{MHz}$ and the video-recording rate was increased to $50000 \mathrm{fps}$. Figure 12 shows an example of acoustic response to formation of the primary deformation band, consisting of two pulses with microsecond duation. It is synchronized in Fig. 13 with the results of processing of video records of the embryo band growth (cf. Fig. 4).

As can be seen from Fig. 13, the first microsecond AE pulse coincides with a small jump in the band tip velocity (frame 1109) with an amplitude of $\sim 3 \mathrm{~m} / \mathrm{s}$ and indicates the onset of the final accelerated stage of growth of the embryo deformation band. The second pulse corresponds with an accuracy of not more than $20 \mu$ s to the moment of impact interaction of the band tip with the lateral surface of the sample. The duration of the leading front of the second pulse is not longer than $0.6 \mu \mathrm{s}$, and the amplitude is almost 5 times the amplitude of the first pulse. According to the video record, the band tip reaches the lateral surface at the instant corresponding to frame 1115 , which gives an estimate of $33 \mathrm{~m} / \mathrm{s}$ for the maximum tip velocity. Judging by the acoustic signal, the second high-amplitude AE pulse, presumably associated with the impact of the band tip on the side surface, is excited at the time between frames 1114 and 1115. If this circumstance is considered, the estimate of the maximum tip velocity should be doubled and will reach at least $60 \mathrm{~m} / \mathrm{s}$.

Unexpectedly, the acoustic noise level drops by more than an order of magnitude in the interval of $220 \mu \mathrm{s}$ between the two pulses with microsecond duration. The noise is restored to the initial level after the end of the second high-amplitude AE pulse (see Fig. 12). According to the above correlation of the microsecond pulses with specific stages of the deformation band formation, such an "internal" absorption of intrinsic acoustic noise or, more precisely, of the continuous AE component may be associated with the generation of a large number of mobile dislocations at the accelerated stage of growth of the incomplete deformation band.

\section{On the mechanisms of nucleation and formation of deformation bands}

As noted above, the embryo band tip grows with acceleration at the final stage of its evolution, reaching the maximum velocity immediately before the instant of impact interaction with the opposite lateral surface of the flat sample. This instant also corresponds to the maximum amplitude of the AE pulse (see also Ref. [30]). The main questions concerning the band growth mechanism involve the following aspects: the spatial coupling mechanism responsible for the band growth, the mobility of dislocations within the band, the effect of the band and the sample 
geometries, the stress concentration at the band tip, especially near the exit of the embryo band on the opposite surface, the impact interaction of its tip with the surface.

The transfer of the dislocation glide over distances of tens and hundreds of microns implies its activation by long-range stress fields. Among various spatial coupling mechanisms discussed in the literature in this respect, the main role is generally attributed to the mechanisms considering slip activation by excess shear stresses at the front of the deformation band [53-55]. As shown in [42], such spatial coupling may be expressed by an additional diffusion-like term in the constitutive equation, proportional to the strain field Laplacian $\nabla^{2} \varepsilon$, which relates the flow stress $\sigma$ to the plastic strain $\varepsilon$ and strain rate $\dot{\varepsilon}[4,56]$.

The most common cause of the excess stress at the front of a deformation band is an elastic accommodation of plastic strain incompatibility at the boundary between the plastically deformed and undeformed regions of the material. Obviously, the maximum incompatibility stresses occur in the region of the maximum Laplacian of the strain field, i.e., near the tip of the needle-shaped embryo band. The stress concentration increases as the band tip approaches the opposite lateral surface of the sample, thus causing an accelerated growth of the embryo band at the final stage of its evolution. According to [4], the additional stress at the moving band front may be expressed as

$$
\tau_{f r}=h l^{2} \nabla_{x}^{2} \varepsilon
$$

where $h$ is the work-hardening coefficient, $l$ is the characteristic length of plastic flow heterogeneity, and $x$-axis corresponds to the tension direction. In polycrystals, the incompatibility stress between neighboring grains in the heterogeneous strain region gives rise to an additional term with a factor $G d^{2} / 4$ before the Laplacian, where $d$ is the average grain size and $G$ is the shear modulus [53]. This expression allows for an estimate of the characteristic length,

$$
l=d(G / 4 h)^{1 / 2}
$$

Taking $G=28 \mathrm{GPa}, d \approx 10 \mu \mathrm{m}$ and $h \approx 2.5 \mathrm{GPa}$ for the initial stage of development of the first deformation band in the studied AlMg6 alloy, this formula renders $l \approx 17 \mu \mathrm{m}$. The obtained value is consistent with the width of the band front, according to the results of computer processing of digital images of the growing bands.

This consideration allows for a discussion of dislocation mechanisms of nucleation and formation of deformation bands. According to the above estimates, the source of a new deformation band occurring at the boundary of the previous band is located within one or several grains. The volume $V_{b}$ of the embryo band increases by a factor of $V_{b} / V_{g} \sim 10^{6}$ during its evolution, where $V_{b} \approx L_{b} w_{b} \delta \approx 1 \mathrm{~mm}^{3}$ at the end of the band transversal growth, the sample thickness $\delta=0.5 \mathrm{~mm}, L_{b}$ and $w_{b}$ are respectively the band length and width, and the grain volume $V_{g} \approx d^{3} \approx 10^{-6} \mathrm{~mm}^{3}$. Therefore, avalanche-like multiplication of dislocations must be the dominant (although possibly not the only one) mechanism of evolution of the band. It can be viewed as follows. When a dislocation source of Frank-Read (F-R) type commonly considered for $f c c$ metals is actuated in the parent grain near the sample surface, it results in the formation of a dislocations pile-up that is blocked by the grain boundary. The arresting of the dislocations creates a positive feedback because the deceleration of the pile-up near the grain boundary gives rise to an overstress pulse and local heating which stimulate the activation of F-R sources in the neighboring grains and may result in a "chain explosion" of the number of fresh dislocation pileups. It might be noted that nonlinear equations of chain reactions are similar to equations 
describing the dynamics of populations [57], which role is played in the present case by the population of mobile dislocations.

It is known that the energy released during an $\mathrm{AE}$ event is approximately proportional to the source volume [58]. Consequently, the energy $E=\int A^{2} d t$ of the acoustic burst generated by the embryo band should be at least six orders of magnitude higher than the AE energy from the F-R source in the parent grain. Therefore, the moment of the band initiation is not detected by the AE method. The expected signal is much lower than the acoustic noise level occurring under given deformation conditions and consisting of the testing machine noise and the continuous $\mathrm{AE}$ component from the plastically deforming sample volume outside the growing embryo band. The major part of acoustic energy is released at the final accelerated stage of embryo band growth, which is characterized by the maximum growth rate of the band volume, $\dot{V}_{b}=\dot{S}_{b} \delta \approx 5 \mathrm{~cm}^{3} / \mathrm{s}$ (see Fig.13, curve d).

The high values of the band growth rate raise a question of the dislocation mobility that is determined by the characteristic dislocation velocities in the embryo band. These velocities cannot be lower than the displacement rate of the band boundary. Therefore, the latter can serve as a rough estimate of the dislocation velocity at the boundary. The lateral growth rate $v_{l}$ usually does not exceed $\sim 10 \mathrm{~cm} / \mathrm{s}$, which corresponds to the thermally activated motion of dislocations and admits the DSA mechanism [4]. At the same time, the tip velocity significantly exceeds the lateral velocity. It is thus reasonable to suggest that when the tip velocity of an embryo band exceeds $10 \mathrm{~m} / \mathrm{s}$, the lattice dislocations move in a quasi-viscous manner within the grain, i.e., perform an over-barrier motion and are not susceptible to DSA. As a result, the tip moves much faster than the lateral boundary, and the shape of the embryo band quickly "stretches" into a narrow wedge in the shape of a semi-ellipse [30].

It can also be suggested that the transition from long (up to $\sim 10 \mathrm{~ms}$ ) broadband acoustic bursts to short series of microsecond AE pulses, which takes place with an increase in the creep stress in the region of $\sim 270-310 \mathrm{MPa}$, is caused by an increase in the velocity of the embryo band tip from $\sim 3 \mathrm{~m} / \mathrm{s}$ to $\sim 30-40 \mathrm{~m} / \mathrm{s}$ and, accordingly, by a transition from the thermally activated to quasi-viscous regime of motion of dislocations. It is known that the latter is determined by the rate of dissipation of the energy of mobile dislocations into various subsystems of the deforming metallic material: phonons, electrons, etc. [59]. A significant part of energy is spent to excite vibrational degrees of freedom of impurity atoms and dislocation segments in various microstructures containing dislocations, e.g., ensembles of fresh dislocations not aged by impurity atoms, grain boundaries, cells, etc.

In this connection, a particular interest is attracted by the result presented at the end of the previous section and manifested as a selective attenuation of acoustic vibrations (at least, at frequencies below $1 \mathrm{MHz}$ ) in an interval of $\sim 200 \mu$ s between the microsecond $\mathrm{AE}$ pulses recorded during the accelerated growth of the embryo band. A hint to the understanding of this peculiarity may be found in Ref. [60] reporting on a strong damping (by more than an order of magnitude) of internal friction during strain jumps in constant stress-rate tensile tests realized on several alloys. Although the authors did not suggest a specific model to explain this phenomenon, the data of experiments clearly relate the high damping coefficient to a high density of mobile dislocations newly formed during formation of deformation bands. This suggestion qualitatively agrees with the strong absorption of the intrinsic acoustic noise found in the present work. Even if the observations in [60] correspond to a distinct frequency range $(\sim 10$ $\mathrm{Hz}$ ), so that specific mechanisms may be qualitatively different, it can be suggested that the observed attenuation is also related to the abrupt dislocation multiplication associated with dynamic reconstructions in the medium carrying the acoustic waves, e.g., through dissipation of the sound energy for activation of new dislocations or through self-damping because of sound reflections from dynamic interfaces associated with strain localizations. It is also noteworthy that the high attenuation coefficient may not apply to the entire volume of the sample but only to the embryo band at the final accelerated growth stage, due to generation of a large number of mobile 
dislocations switching to the quasi-viscous mode of motion. The mechanism of this phenomenon is an open question and requires further investigation.

\section{SUMMARY}

The discontinuous creep resulting from spontaneous nucleation and growth of deformation bands at a constant applied stress presents a striking example of nonlinear dynamics, which might serve as a model of the macroscopic-scale self-organization in nonlinear systems studied in various fields of science. Self-organized deformation processes giving rise to plastic instabilities have been attracting strong interest of researchers since the mid-eighties of the last century, but most of the research was aimed at relatively large time scales, often corresponding to the duration of a mechanical test. In the present paper, the millisecond dynamics of embryo deformation bands was investigated experimentally using the AE technique simultaneously with high-speed videorecording of the surface of the deforming samples of an AlMg6 alloy. The duration of the embryo band growth usually does not exceed several milliseconds. Therefore, an experimental study of this process requires the use of methods operating in a frequency band significantly exceeding $1 \mathrm{kHz}$. In the present work, data on the deformation band formation were obtained by virtue of combination of the AE method covering a frequency band from $25 \mathrm{kHz}$ to $\sim 1 \mathrm{MHz}$ and high-speed video-recording at a rate of 5000 to $50000 \mathrm{fps}$ with a spatial resolution of 10 to 40 $\mu \mathrm{m} /$ pixel.

The experiments permit the following conclusions to be drawn:

(i) Embryo bands in flat tensile samples can be classified into four main types with regard to their geometry and dynamics: primary bands, growing across the frontal surface in the direction of the maximum tangential stresses; secondary bands, growing along the border of the previous band; secondary conjugated bands, intersecting with the previous bands at an angle of about $60^{\circ}$; and arcuate bands, growing in the area of the sample heads. The fastest dynamics is characteristic of the first two types, while the band of the fourth type is the slowest of all at the same level of the applied load. All types of embryo bands show nonlinear dynamics with a sharp acceleration at the final stage of growth. They generate bell-shaped acoustic bursts characterized by a broadband $(\sim 25 \mathrm{kHz}-1 \mathrm{MHz})$ power spectrum, various amplitudes, and durations in the range of $\sim 1-10 \mathrm{~ms}$, depending on the band type and the applied stress in the range below $\sim 0.8 \sigma_{u}$ (here, $\sigma_{u}$ is the ultimate tensile strength). At higher stresses, the bands of the first and second type generate short, lasting up to $\sim 1 \mathrm{~ms}$, series of high-amplitude microsecond AE pulses.

(ii) It is established that the sources of broadband AE bursts are related to the final stage of the embryo band growth, which consists of the impact interaction of its tip with the surface, accounting for the maximum amplitude of the acoustic burst, and the subsequent stage of the band expansion, corresponding to the signal attenuation. The power spectrum of broadband bursts contains virtually all frequencies of the studied range with the main maximum near 100 $\mathrm{kHz}$ and the median frequency about $300 \mathrm{kHz}$. The shape of the averaged envelope of the AE burst correlates well with the absolute rate of growth of the force response to the deformation band formation.

(iii) The broadband acoustic burst caused by the formation of an individual band has a complex fractal structure consisting of a large number of short $(\sim 1-10 \mu \mathrm{s})$ discrete AE pulses with a power-law amplitude distribution. Such scale-invariant statistics are usually considered as a signature of avalanche-like dynamics of the underlying physical processes. It may be supposed that dislocation avalanches are the building blocks of the growing deformation band. It should be underlined that the statistical analyses reported in the literature correspond to time intervals of 
minutes and even hours, within which millisecond pulses analyzed in the present paper would be represented by structureless points. This similitude between behaviors on distinct time scales motivated fractal analysis that revealed a multifractal nature of broadband acoustic bursts and corroborated the conjecture of self-organized dynamics of dislocation avalanches over the time scale of a deformation band.

(iv) Short series of microsecond AE pulses detect with an accuracy of $\sim 1 \mu$ s the stage of accelerated growth of the first and second type embryo bands occurring at high stresses. The first $\mathrm{AE}$ pulse in the series indicates the onset of this stage, and the AE pulse with the maximum amplitude reveals the shock interaction of the embryo band tip with the sample surface. According to video-recording at a rate of $50000 \mathrm{fps}$, the tip speed can reach $\sim 30-40 \mathrm{~m} / \mathrm{s}$ at this instant.

(v) The source of a deformation band lies within one or several neighboring grains. The volume of the embryo band increases by more than six orders of magnitude during its growth. It can thus be suggested that the avalanche-like multiplication of dislocations is the dominant growth mechanism. At the stage of accelerated growth, dislocations move in a quasi-viscous regime in the embryo band tip, and the lateral growth occurs due to thermally activated motion of dislocations. A surprising observation concerns an unexpectedly strong attenuation (by more than an order of magnitude) of acoustic noise at the stage of accelerated growth. Several hypotheses associated with the generation of a large number of mobile dislocations are suggested as possible mechanisms of such phenomenon. The verification of these conjectures will require further investigations.

The experimental data connote several remarks concerning mechanical properties and applications of alloys prone to plastic instability and strain localization. First, attention should be attracted to the fact that the source of a deformation band lies within one, perhaps, several polycrystalline grains. It will be of great interest to extend this study to similar alloys with various grain size, including submicro- or nanocrystalline materials or samples with grains refined in a surface layer that might control the embryo band nucleation. It is noteworthy in this context that efficient suppression of the PLC effect was observed after severe plastic deformation of some AlMg alloys in [61, 62]. Second, the observation of a radical change in the waveform of AE signal on approaching the ultimate stress may serve to anticipate the oncoming fracture. Moreover, the knowledge of the correlation between AE bursts and the formation of deformation bands may help to find original ways to control or suppress strain localization and, therefore, improve and stabilize mechanical properties of alloys. For example, recent studies showed that application of electrical current can suppress the PLC effect and band formation [63]. In this case, AE signals may provide feedback to control electric current generators.

\section{Acknowledgements}

The authors are grateful to G.A. Malygin for attracting their attention to a research on the effect of strain localization on internal friction in alloys. The present research was supported by the Russian Science Foundation (project No. 18-19-00304) and the Russian Foundation for Basic Research (project No. 19-08-00395) with the use of equipment of the Center of Collective Use of Tambov State University. M.L. and T.L M.L. gratefully acknowledges support from the French State through the program "Investment in the future" operated by the National Research Agency, in the framework of the LabEx DAMAS [ANR-11-LABX-0008-01] and the research program RESEM managed by the Institut de Recherche Technologique en Matériaux, Métallurgie et Procédés (IRT M2P). 


\section{References}

1. F. Savart, Recherches sur les vibrations longitudinales, Ann. Chim. Fhys. 65, 337 (1837).

2. E.N. da C. Andrade, On the viscous flow in metals and allied phenomena, Proc. R. Soc. A 84, 1 (1910).

3. A. Portevin, and F. Le Chatelier, Sur un phénomène observé lors de l'essai de traction d'alliages en cours de transformation, C. R. Acad. Sci. Paris. 176, 507 (1923).

4. Y. Estrin, and L.P. Kubin, Spatial coupling and propagative plastic instabilities, in Continuum models for materials with microstructure, Edited by H.-B. Muhlhaus (Wiley \& Sons, New York, 1995), pp. 395-450.

5. F. Heslot, T. Baumberger, B. Perrin, B. Caroli, and C. Caroli, Creep, stick-slip, and dry-friction dynamics - experiments and a heuristic model, Phys. Rev. E 49, 4973 (1994). https://doi.org/10.1103/PhysRevE.49.4973

6. J.M. Carlson, J.S. Langer, and B.E. Shaw, Dynamics of earthquake faults, Rev. Mod. Phys. 66, 657 (1994). https://doi.org/10.1103/RevModPhys.66.657

7. R. Livermore, The tectonic plates are moving! Oxford Scholarship Online, 2018. https://doi.org/10.1093/oso/9780198717867.001.0001

8. L.P. Kubin, C. Fressengeas, and G. Ananthakrishna, Collective behaviour of dislocations in plasticity, Dislocations in Solids 11, 101 (2002).

9. G. Ananthakrishna, Current theoretical approaches to collective behavior of dislocations, Phys. Rep.Rev. Sec. Phys. Lett. 440, 113 (2007). https://doi.org/10.1016/j.physrep.2006.10.003

10. M. A. Lebyodkin, T. A. Lebedkina, and A. Jacques, Multifractal analysis of unstable plastic flow (Nova Science Publishers, Hauppauge, NY, 2009).

11. K. Chihab, Y. Estrin, L.P. Kubin, and J. Vergnol, The kinetics of the Portevin-Le Chatelier bands in an Al-5 at\%Mg alloy, Scr. Metall. 21, 203 (1987). https://doi.org/10.1016/0036-9748(87)90435-2

12. A.A. Shibkov, M.F. Gasanov, M.A. Zheltov, A.E. Zolotov, and V.I. Ivolgin, Intermittent plasticity associated with the spatio-temporal dynamics of deformation bands during creep tests in an AlMg polycrystal, Int. J. Plast. 86, 37 (2016). https://doi.org/10.1016/j.ijplas.2016.07.014.

13. R.B. Schwarz, and L.L. Funk, Kinetics of the Portevin-Le Chatelier effect in Al 6061 alloy, Acta metal. 33, 295 (1985). https://doi.org/10.1016/0001-6160(85)90148-8

14. A. Yilmaz, The Portevin-Le Chatelier effect: a review of experimental findings, Sci. Thechnol. Adv. Mater. 12, 063001 (2011). https://doi.org/10.1088/1468-6996/12/6/063001

15. T.A. Lebedkina, Y. Bougherira, D. Entemeyer, M.A. Lebyodkin, and I.V. Shashkov, Crossover in the scale-free statistics of acoustic emission associated with the Portevin-Le Chatelier instability, Scr. Mater. 148, 47 (2018). DOI: 10.1016/j.scriptamat.2018.01.017

16. T.A. Lebedkina, D.A. Zhemchuzhnikova, and M.A. Lebyodkin, Correlation versus randomization of jerky flow in an AlMgScZr alloy using acoustic emission, Phys. Rev. E 97, 013001 (2018). https://doi.org/10.1103/PhysRevE.97.013001

17. M.A. Lebyodkin, N.P. Kobelev, Y. Bougherira, D. Entemeyer, C. Fressengeas, V.S. Gornakov, T.A. Lebedkina, and I.V. Shashkov, On the similarity of plastic flow processes during smooth and jerky flow: Statistical analysis, Acta Mater. 60, 3729 (2012). https://doi.org/10.1016/j.actamat.2012.03.026.

18. P. Bak, C. Tang, and K. Wiesenfeld, Self-organized criticality, Phys. Rev. A 38, 364 (1988). https://doi.org/10.1103/PhysRevA.38.364

19. H.D.I. Abarbanel, R. Brown, J.J. Sidorowich, and L.Sh. Tsimring, The analysis of observed chaotic data in physical systems, Rev. Mod. Phys. 65, 1331 (1993). https://doi.org/10.1103/RevModPhys.65.1331

20. S.H. Strogatz, From Kuramoto to Crawford: Exploring the onset of synchronization in populations of coupled oscillators. Phys. D 143, 1 (2000). https://doi.org/10.1016/S0167-2789(00)00094-4

21. M. Lebyodkin, Y. Bougherira, T. Lebedkina, and D. Entemeyer, Scaling in the Local Strain-Rate Field during Jerky Flow in an Al-3\%Mg Alloy, Metals 10, 134 (2020). https://doi.org/10.3390/met10010134

22. F.B. Klose, F. Hagemann, P. Hähner, and H. Neuhäuser, Investigation of the Portevin-Le Chatelier effect in Al-3wt.\%Mg alloys by strain-rate and stress-rate controlled tensile tests, Mater. Sci. Eng. A 387-389, 93 (2004). https://doi.org/10.1016/j.msea.2004.01.062 
23. G.F. Xiang, Q.C. Zhang, H.W. Liu, X.P. Wu, and X.Y. Ju, Time-resolved deformation measurements of the Portevin-Le Chatelier bands, Scr. Mater. 56, 721 (2007). https://doi.org/10.1016/j.scriptamat.2006.08.049

24. M.M. Krishtal, A.K. Khrustalev, A.V. Volkov, and S.A. Borodin, Nucleation and growth of macrofluctuations of plastic strain with discontinuous yield and luders deformation: Results of highspeed video filming, Dokl. Phys. 54, 225 (2009). https://doi.org/10.1134/S1028335809050024

25. L. Casarotto, H. Dierke, R. Tutsch, and H. Neuhäuser, On nucleation and propagation of PLC bands in an Al-3Mg alloy, Mater. Sci. Eng. A 527, 132 (2009). https://doi.org/10.1016/j.msea.2009.07.043

26. W. Tong, H. Tao, N. Zhang, and L.G. Hector Jr., Time-resolved strain mapping measurements of individual Portevin-Le Chatelier deformation bands, Scr. Mater. 53, 87 (2005) https://doi.org/10.1016/j.scriptamat.2005.03.020

27. J. Zdunek, T. Brynk, J. Mizera, Z. Pakiela, and K. J. Kurzydlowski, Digital image correlation investigation of Portevin-Le Chatelier effect in an aluminium alloy, Mater. Charact. 59, 1429 (2008). https://doi.org/10.1016/j.matchar.2008.01.004

28. M.A. Lebyodkin, D.A. Zhemchuzhnikova, T.A. Lebedkina, and E.C. Aifantis, Kinematics of formation and cessation of type B deformation bands during the Portevin-Le Chatelier effect in an AlMg alloy, Results Phys. 12, 867 (2019). https://doi.org/10.1016/j.rinp.2018.12.067

29. A.A. Shibkov, M.A. Zheltov, M.F. Gasanov, and A.E. Zolotov, Mechanism of low-frequency discrete acoustic emission during intermittent creep of aluminum alloy, Phys. Solid State 59, 2387 (2017). https://doi.org/10.1134/S1063783417120289

30. A.A. Shibkov, M.A. Zheltov, M.F. Gasanov, A.E. Zolotov, A.A. Denisov, and M.A. Lebyodkin, Dynamics of deformation band formation investigated by high-speed techniques during creep in an AlMg alloy, Mater. Sci. Eng. A 772, 138777 (2020). https://doi.org/10.1016/j.msea.2019.138777

31. M.A. Lebyodkin, T.A. Lebedkina, F. Chmelik, T.T. Lamark, Y. Estrin, C. Fressengeas, and J. Weiss, Intrinsic structure of acoustic emission events during jerky flow in an Al alloy, Phys. Rev. B 79, 174114-9 (2009). https://doi.org/10.1103/PhysRevB.79.174114

32. A.A. Shibkov, and A.E. Zolotov, Nonlinear dynamics of the spatio-temporal pattern of a macroscopically localized deformation, JETP Lett. 90, 370 (2009). https://doi.org/10.1134/S0021364009170123.

33. L.S. Derevyagina, V.E. Panin, and A.I. Gordienko, Self-organization of plastic shears in localized deformation macrobands in the neck of high-strength polycrystals and its role in material fracture under uniaxial tension, Phys. Mesomech. 11,51 (2008). https://doi.org/10.1016/j.physme.2008.03.006

34. D. Yuzbekova, A. Mogucheva, D. Zhemchuzhnikova, T. Lebedkina, M. Lebyodkin, and R. Kaibyshev, Effect of microstructure on continuous propagation of the Portevin-Le Chatelier deformation bands, Int. J. Plast. 96, 210 (2017). https://doi.org/10.1016/j.ijplas.2017.05.004

35. D. Zhemchuzhnikova, M. Lebyodkin, D. Yuzbekova, T. Lebedkina, A. Mogucheva, and R. Kaibyshev, Interrelation between the Portevin-Le Chatelier effect and necking in AlMg alloys, Int. J. Plast. 110, 95 (2018). https://doi.org/10.1016/j.ijplas.2018.06.012

36. A.H. Cottrell, Theory of brittle fracture in steel and similar metals, Trans. Met. Soc. AIME. 212, 192 (1958).

37. F. Chmelik, A. Ziegenbein, H. Neuhauser, and P. Lukac, Investigating the Portevin-Le Chatelier effect by the acoustic emission and laser extensometry techniques, Mater. Sci. Eng. A 324, 200 (2002). https://doi.org/10.1016/S0921-5093(01)01312-0

38. F. Chmelik, F.B. Klose, H. Dierke, J. Sachl, H. Neuhauser, and P. Lukac, Investigating the PortevinLe Chatelier effect in strain rate and stress rate controlled tests by the acoustic emission and laser extensometry techniques, Mater. Sci. Eng. A 462, 53 (2007). https://doi.org/10.1016/j.msea.2006.01.169

39. I.V. Shashkov, M.A. Lebyodkin, and T.A. Lebedkina, Multiscale study of acoustic emission during smooth and jerky flow in an AlMg alloy, Acta. Mater. 60, 6842 (2012). https://doi.org/10.1016/j.actamat.2012.08.058

40. A. Vinogradov, and A. Lasarev, Continuous acoustic emission during intermittent plastic flow in $\alpha$ brass, Scr. Mater. 66, 745 (2012). https://doi.org/10.1016/j.scriptamat.2012.01.053

41. M.-C. Miguel, A. Vespignani, S. Zapperi, J. Weiss, and J.-R. Grasso, Intermittent dislocation flow in viscoplastic deformation, Nature 410, 667 (2001). https://doi.org/10.1038/35070524

42. J. Weiss, and J.-R. Grasso, Acoustic emission in single crystals of ice, J. Phys. Chem. B. 101, 6113 (1997). https://doi.org/10.1021/jp963157f 
43. J. Weiss, J.-R. Grasso, M.-C. Miguel, A. Vespignani, and S. Zapperi, Complexity in dislocation dynamics: experiments, Mater. Sci. Eng. A. 309-310, 360 (2001). https://doi.org/10.1016/S0921$\underline{\text { 5093(00)01633-6 }}$

44. M.A. Lebyodkin, I.V. Shashkov, T.A. Lebedkina, and V.S. Gornakov, Experimental investigation of the effect of thresholding on temporal statistics of avalanches, Phys. Rev. E. 95, 032910 (2017). https://doi.org/10.1103/PhysRevE.95.032910

45. J. Weiss, W. Ben Rhouma, T. Richeton, S. Dechanel, F. Louchet, and L. Truskinovsky, From Mild to WildLV14378 Fluctuations in Crystal Plasticity, Phys. Rev. Lett. 114, 105504 (2015). https://doi.org/10.1103/PhysRevLett.114.105504

46. D.M. Dimiduk, C. Woodward, R. LeSar, and M.D. Uchic, Scale-free intermittent flow in crystal plasticity. Science 312 (2006) 1188-1190. https://doi.org/10.1126/science.1123889

47. M. Zaiser, Scale invariance in plastic flow of crystalline solids, Adv. Phys. 55 (2006) 185-245. https://doi.org/10.1080/00018730600583514

48. H.J. Jensen, Self-organized criticality (Cambridge University Press, Cambridge, 1998).

49. J. Feder, Fractals (Plenum Press, New York, London, 1988).

50. T.C. Halsey, M.H. Jensen, L.P. Kadanoff, I.Procaccia, and B.I. Shraiman, Fractal measures and their singularities: The characterization of strange sets, Phys. Rev. A 33, 1141 (1986). https://doi.org/10.1103/PhysRevA.33.1141

51. A.B. Chhabra, and R.V. Jensen, Direct determination of the $f(\alpha)$ singularity spectrum, Phys. Rev. Lett. 62, 1327 (1989). https://doi.org/10.1103/PhysRevLett.62.1327

52. M.A. Lebyodkin, and T.A. Lebedkina, Multifractal analysis of evolving noise associated with unstable plastic flow, Phys. Rev. E 73, 036114 (2006). https://doi.org/10.1103/PhysRevE.73.036114

53. P. Hähner, Modelling of propagative plastic instabilities, Scr. Metall. Mater. 29, 1171 (1993).

54. E.C. Aifantis, On the role of gradients in the localization of deformation and fracture, Int. J. Eng. Sci. 30, 1279 (1992). https://doi.org/10.1016/0020-7225(92)90141-3

55. M.A. Lebyodkin, and L.R. Dunin-Barkowskii, Critical behavior and mechanism of strain correlations under conditions of unstable plastic flow, J. Exp. Theor. Phys. 86, 993 (1998). https://doi.org/10.1134/1.558571

56. P. Penning, Mathematics of the Portevin-Le Chatelier effect, Acta Metall. 20, 1169 (1972).

57. A.G. Merzhanov, and E.N. Rumanov, Nonlinear effects in macroscopic kinetics, Sov. Phys. Usp. 30, 293 (1987) 293. http://dx.doi.org/10.1070/PU1987v030n04ABEH002831

58. A.G. Beattie, Acoustic emission non-destructive testing of structures using source location techniques (Sandia National Laboratories, Albuquerque, 2013). https://doi.org 10.2172/1096442.

59. V.I. Alshits, and V.L. Indenbom, Mechanism of dislocation drag, in Dislocations in Solids, edited by F.R.N. Nabarro, Vol. 7 (Elsevier Science Pub. Co, North-Hollandn Amsterdam, 1986) pp. 43-111 .

60. J. Baruch, and S.R. Bodner, Internal friction during repeated discontinuous yielding of metals, Mater. Sci. Eng. 14, 81 (1974). https://doi.org/10.1016/0025-5416(74)90125-6

61. M.V. Markushev, and M.Yu. Murashkin, Structure and mechanical properties of commercial Al-Mg 1560 alloy after equal-channel angular extrusion and annealing, Mater. Sci. Eng. A 367, 234 (2004). https://doi:10.1016/j.msea.2003.10.237

62. T.A. Lebedkina, M.A. Lebyodkin, T.T. Lamark, M. Janeček, and Y. Estrin, Effect of equal channel angular pressing on the Portevin-Le Chatelier effect in an Al3Mg alloy, Mater. Sci. Eng. A 615, 7 (2014). http://dx.doi.org/10.1016/j.msea.2014.07.064

63. A.A. Shibkov, A.A. Denisov, M.A. Zheltov, A.E. Zolotov, and M.F. Gasanov, The electric currentinduced suppression of the Portevin - Le Chatelier effect in Al-Mg alloys, Mater. Sci. Eng. A 610, 338 (2014). https://doi:10.1016/j.msea.2014.05.050 


\section{Figure captions}

Fig. 1. Types of embryo deformation bands: (a) primary band; (b) band growing along the boundary of a previous band; (c) conjugated band growing across the previous bands at an angle of about $60^{\circ}$; (d) arcuate band in the region of the sample shoulder. The upper images are the asrecorded photographs and the lower ones are the result of computer processing of the images of the growing embryo band.

Fig. 2. Typical AE signals generated during the formation of deformation bands: (a) broadband bursts of AE; (b) series of high-amplitude microsecond pulses separated by intervals of lowfrequency damped oscillations.

Fig. 3. Formation of a primary deformation band near the sample head, which serves as a trigger for the development of a strain jump in the AlMg6 alloy under conditions of discontinuous creep at $\sigma_{0} \approx 285 \mathrm{MPa}$. The video recording speed is $5000 \mathrm{fps}$. The frame numbers are indicated on the left of the images.

Fig. 4. Time dependences of various characteristics of the primary deformation band: the band length $L_{b}(\mathrm{a})$; the band tip velocity $v_{t}(\mathrm{~b})$, the band area $S_{b}(\mathrm{c})$, its growth rate $\dot{S}_{b}(\mathrm{~d})$; the AE signal $\varphi_{A E}(\mathrm{e})$.

Fig. 5. Power spectrum $P(f)$ of the AE signal shown in Fig. 4, curve (e).

Fig. 6. Synchronization of the time evolution of several quantities: stress $\sigma$ showing an unloading jump (a), acoustic burst $\varphi_{A E}$ caused by the formation of an individual deformation band (b), the corresponding absolute value $\left|\varphi_{\mathrm{AE}}\right|$ (c), its envelope $\xi(\mathrm{d})$, and the absolute values of the stress change rate $\psi=|\dot{\sigma}|$ (e). $\Delta \xi_{m}$ and $\Delta \psi_{m}$ represent the amplitude of the envelope of the acoustic burst and the jump in the stress change rate, respectively.

Fig. 7. Amplitude $\Delta \xi_{m}$ of the envelope of the AE burst caused by the formation of a single deformation band versus amplitude $\Delta \psi_{m}$ of the rate of change of the stress response.

Fig. 8. Temporal structure of an AE burst caused by the formation of an individual deformation band in the AlMg6 alloy, displayed at different time scales.

Fig. 9. Probability distribution function $D(s)$ of normalized squared amplitudes of discrete AE signals in the structure of an acoustic burst caused by the formation of a separate deformation band: (a) linear coordinates; (b) double logarithmic coordinates.

Fig. 10. Results of R/S analysis showing a dependence of the rescaled R/S range on time interval $\tau$ in double logarithmic coordinates. The slope of the dashed line gives the estimate of the Hurst exponent, $H=0.304 \pm 0.06$.

Fig. 11. Singularity spectrum $f_{D}(\alpha)$ for the same AE signal.

Fig. 12. Shape of the acoustic signal corresponding to the accelerated stage of growth of the primary band embryo at a creep stress $\sigma_{0}=310 \mathrm{MPa}$. Inset shows an AE pulse with a front duration of $0.6 \mu$ s caused by the band exit to the opposite side surface of the sample, according to video recording at the rate of $50000 \mathrm{fps}$. $n$ indicates the frame numbers.

Fig. 13. Correlation of the high-frequency acoustic signal (a) shown in Fig. 12 with the length $L_{b}$ of the embryo of the primary band (b), the tip velocity $v_{t}$ (c), and the rate $\dot{S}_{b}$ of the growth of its area (d). The recording speed is $50000 \mathrm{fps}$. The time interval between frames is of $20 \mu \mathrm{s}$. 
(a)
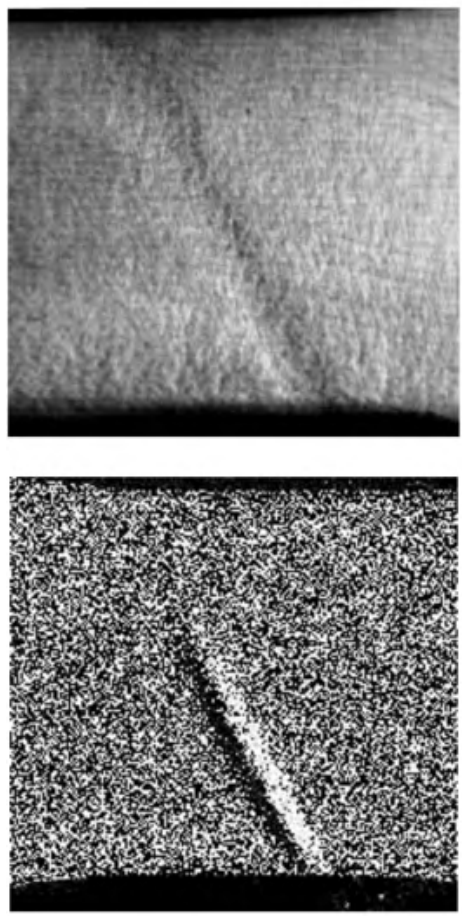

(c)
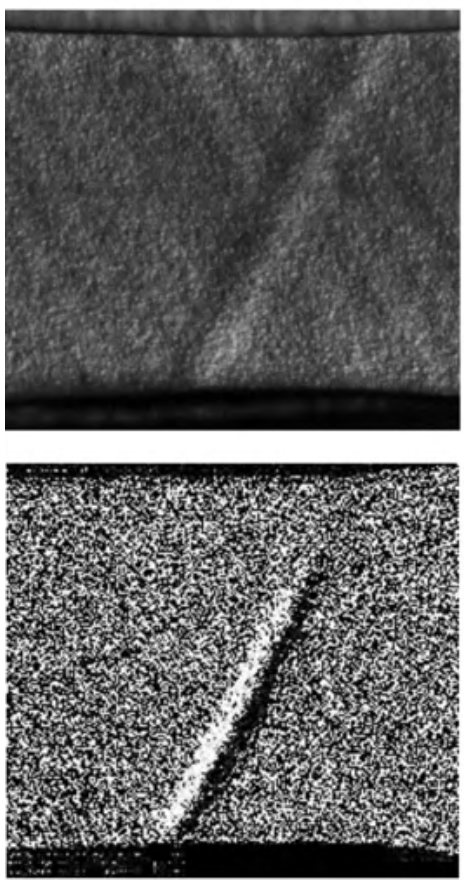

(b)
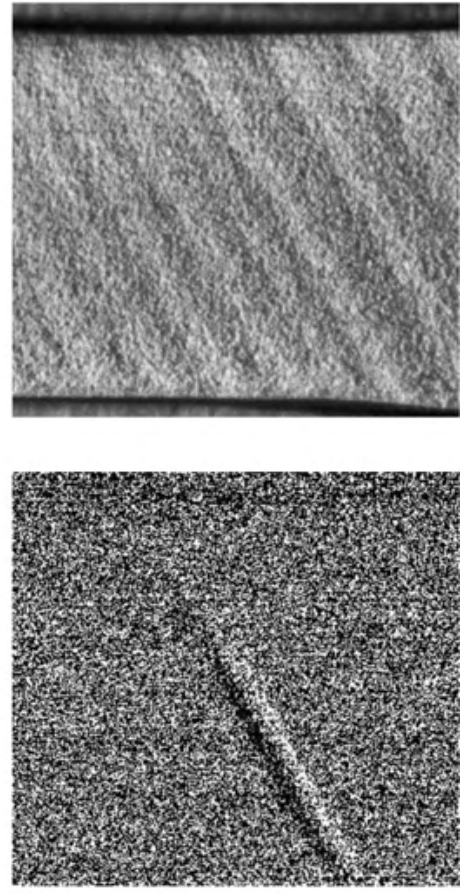

(d)
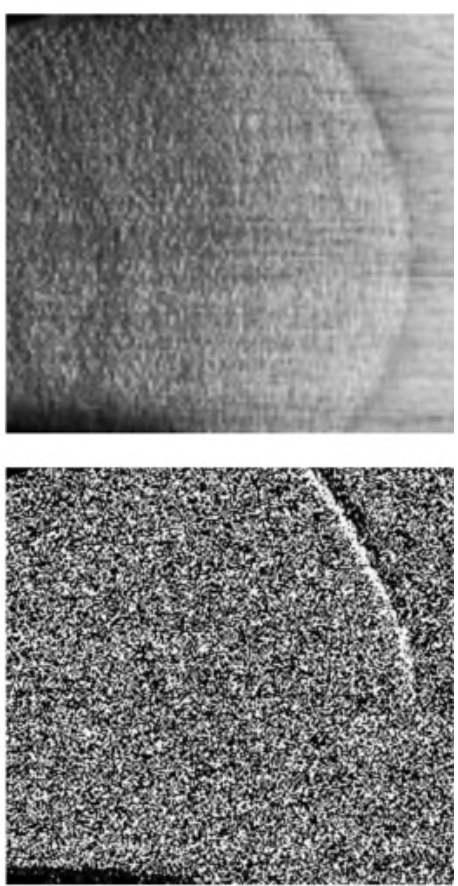

$1 \mathrm{~mm}$

Figure 1 

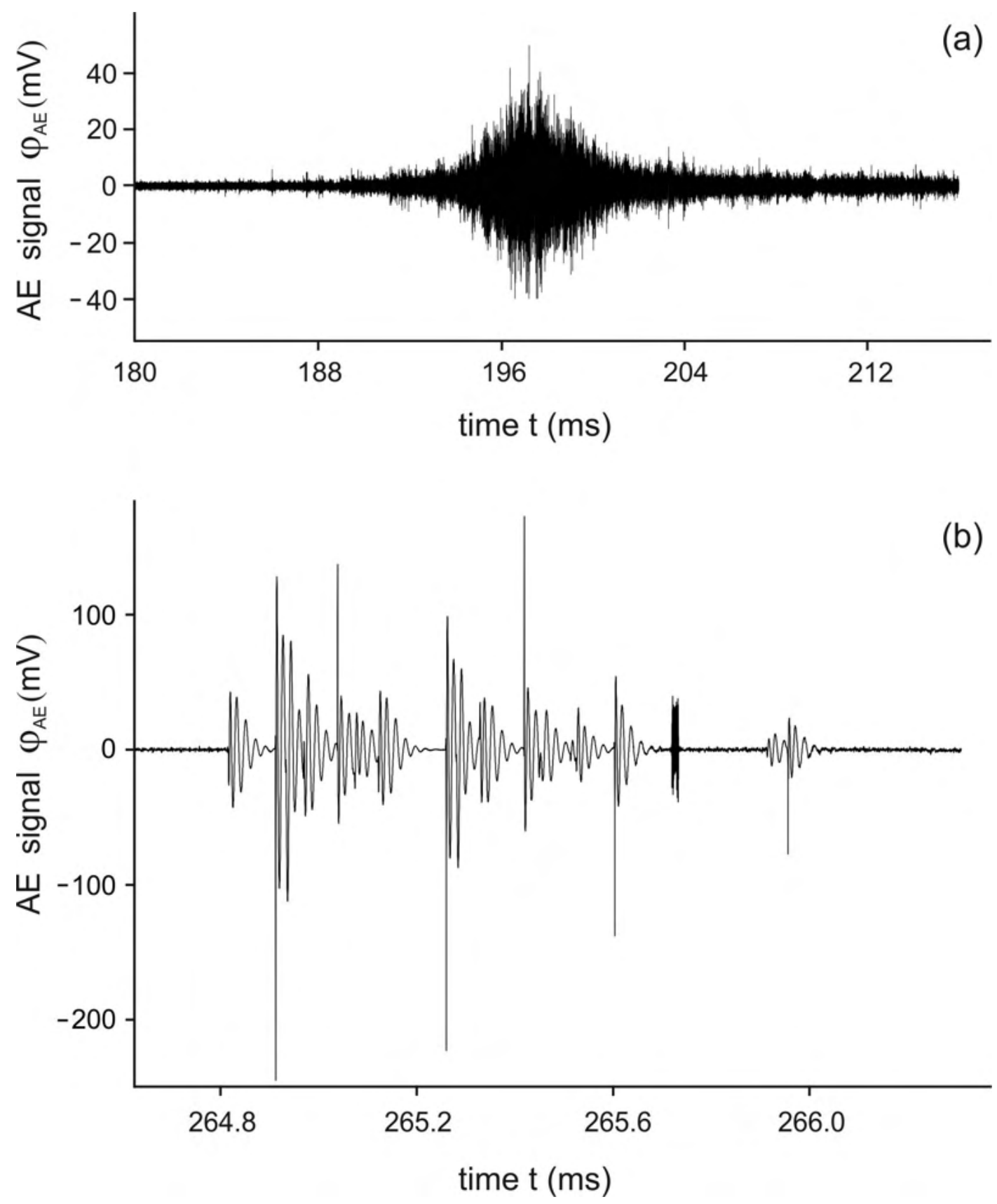

Figure 2 

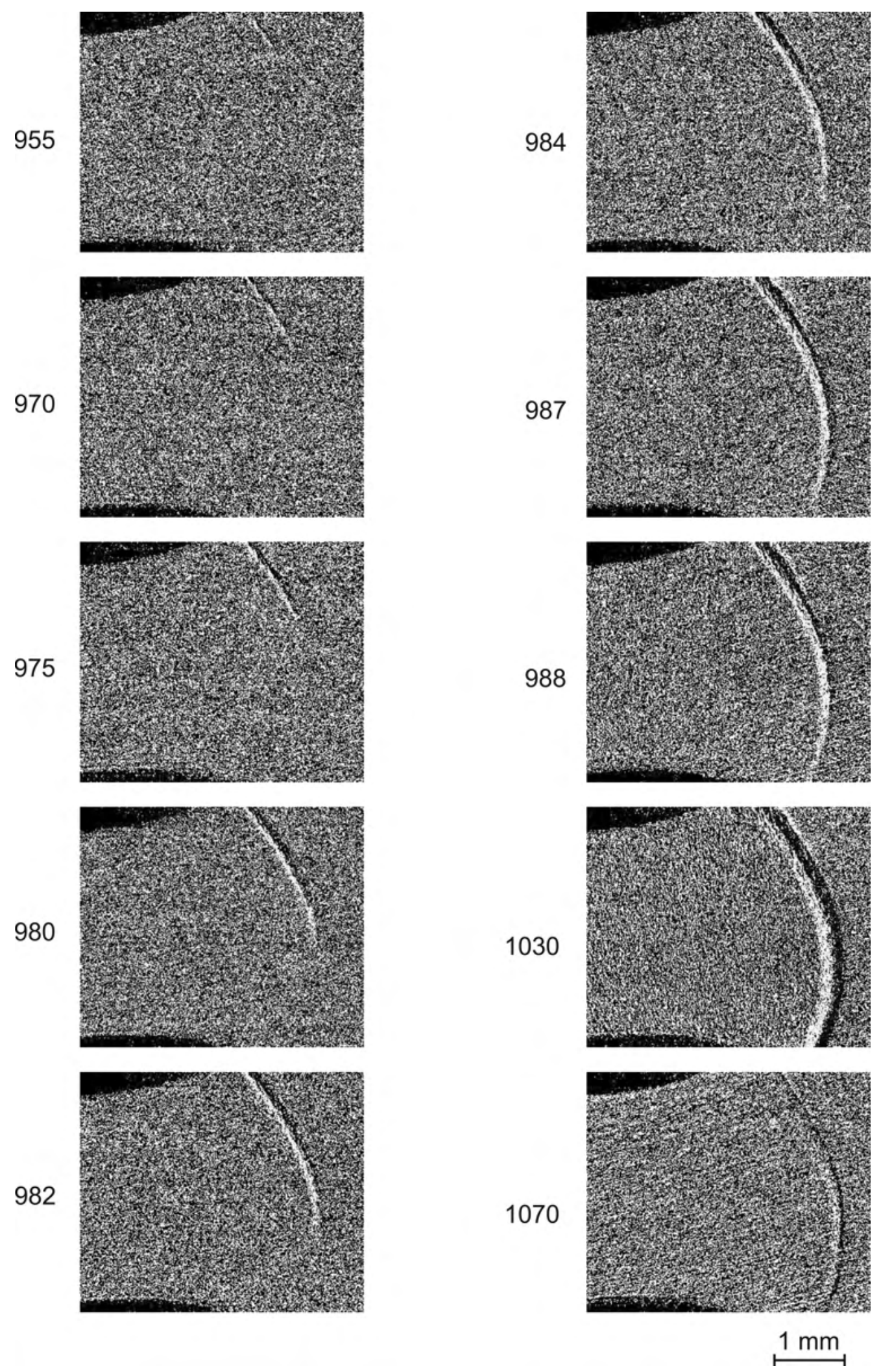

Figure 3 

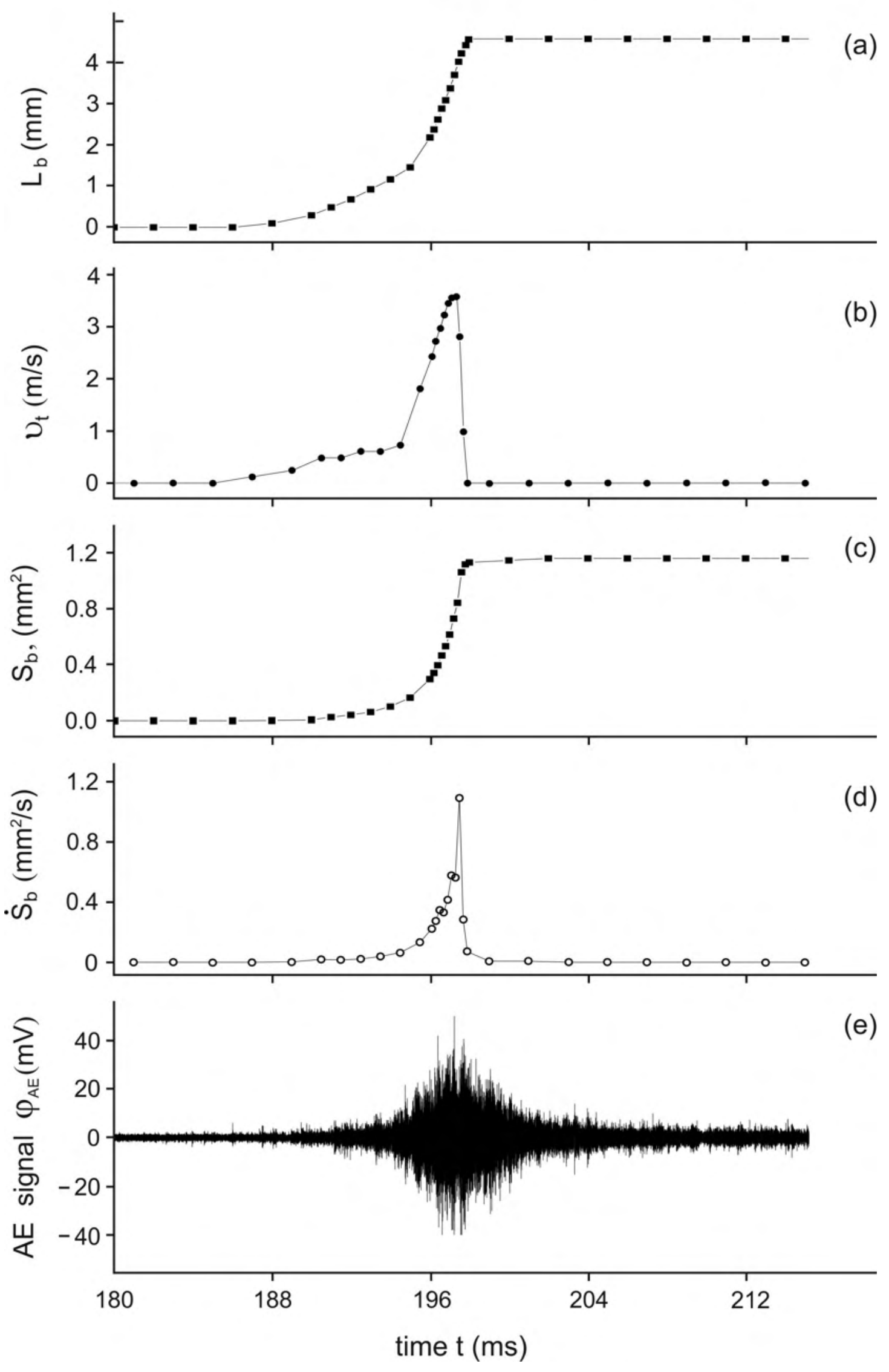

Figure 4 


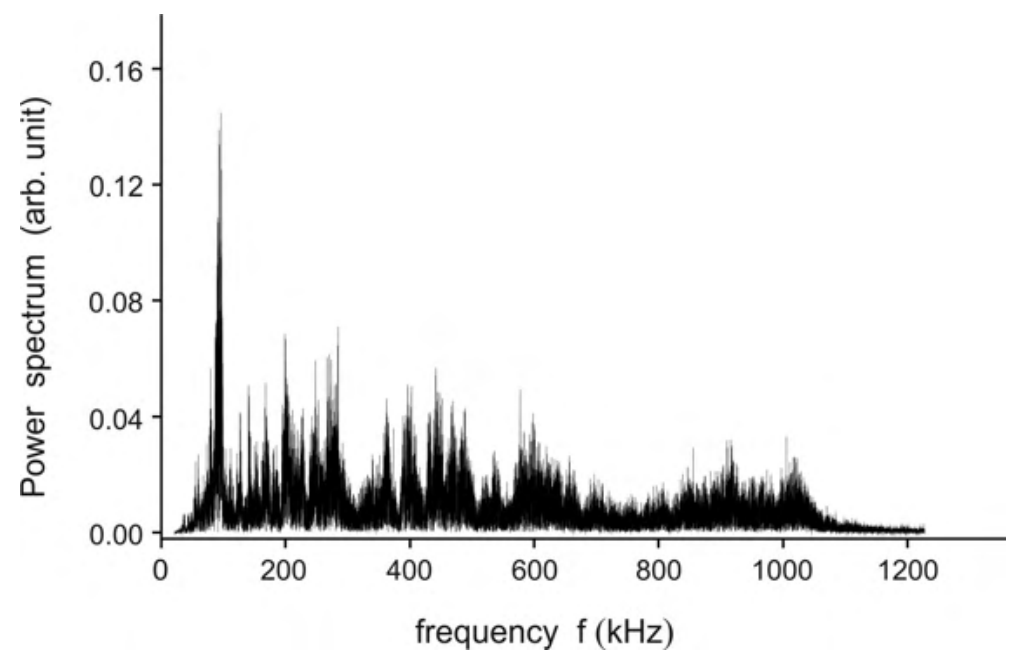

Figure 5
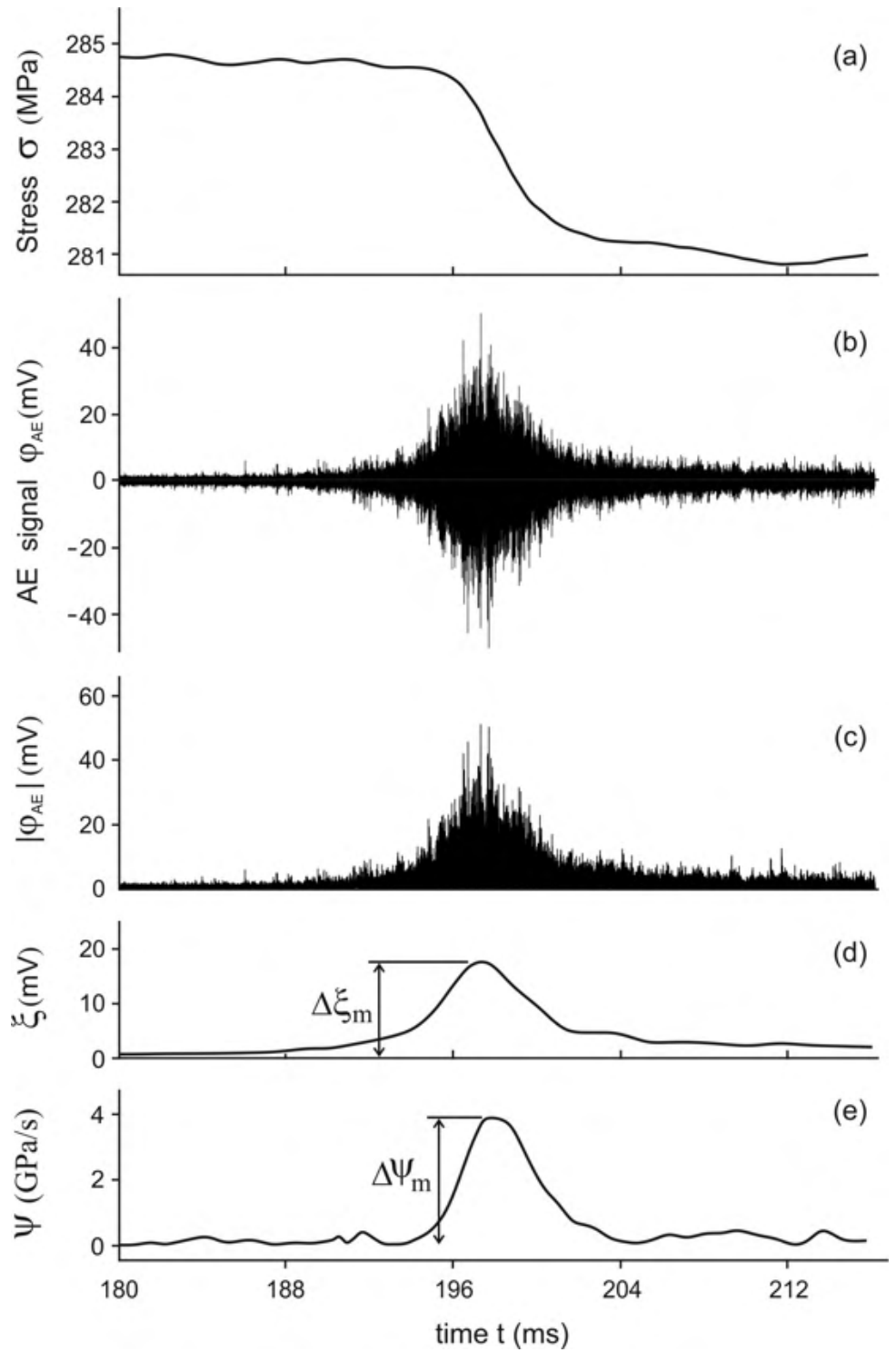

Figure 6 


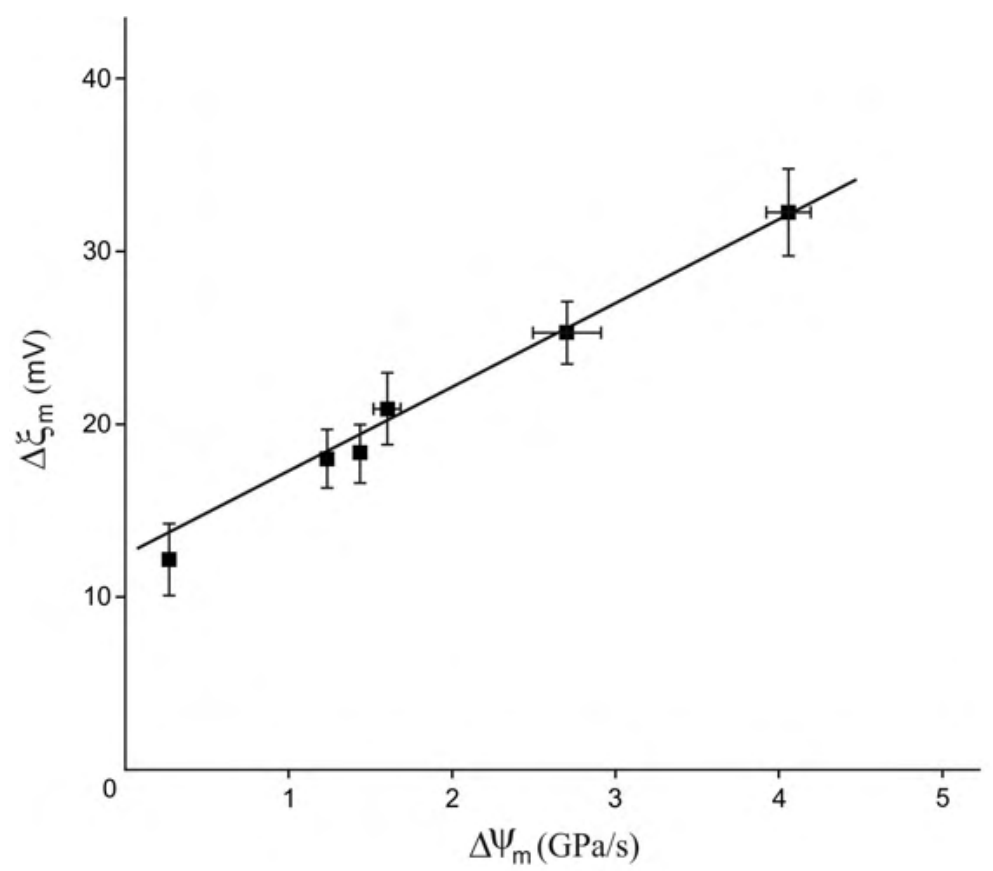

Figure 7

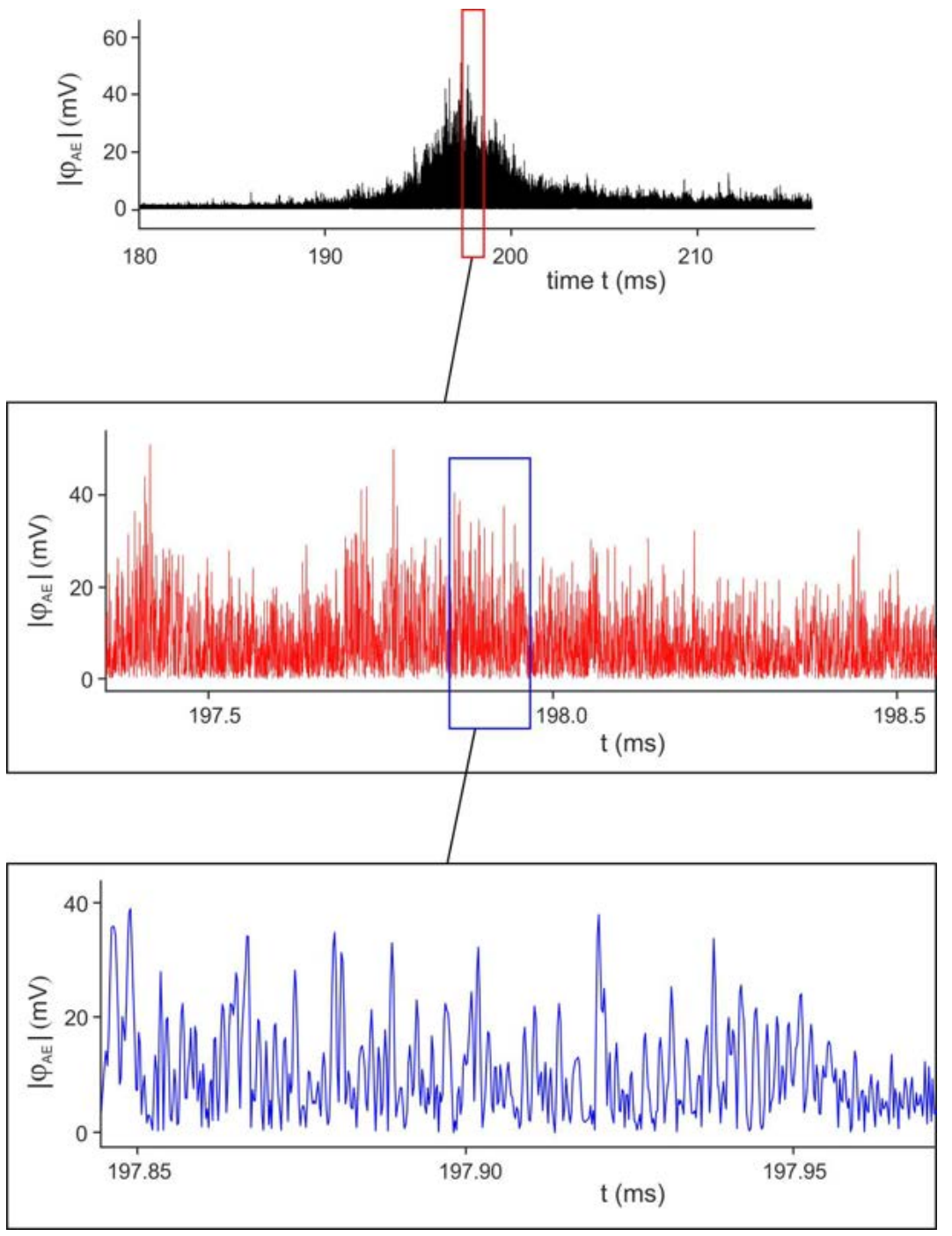

Figure 8 
(a)

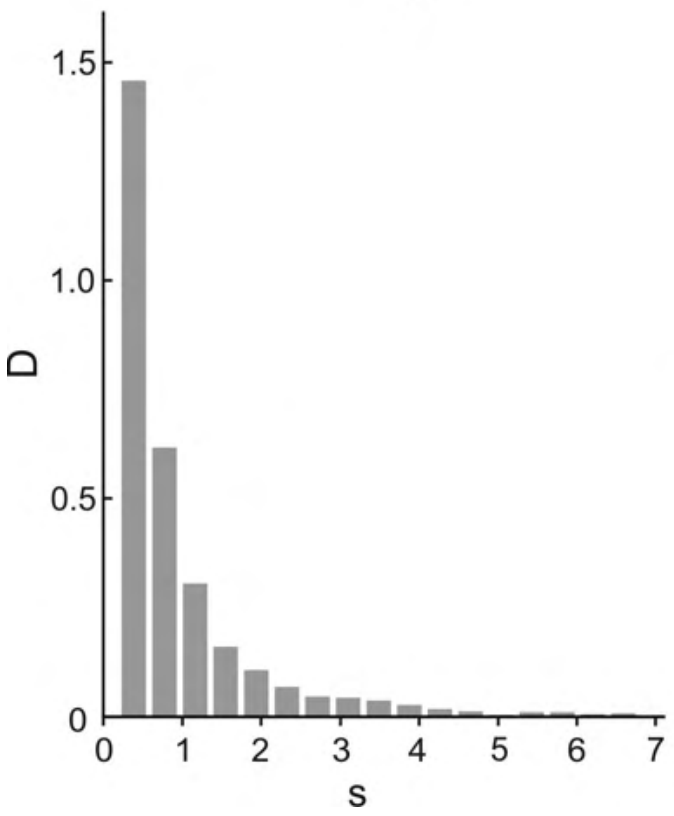

(b)

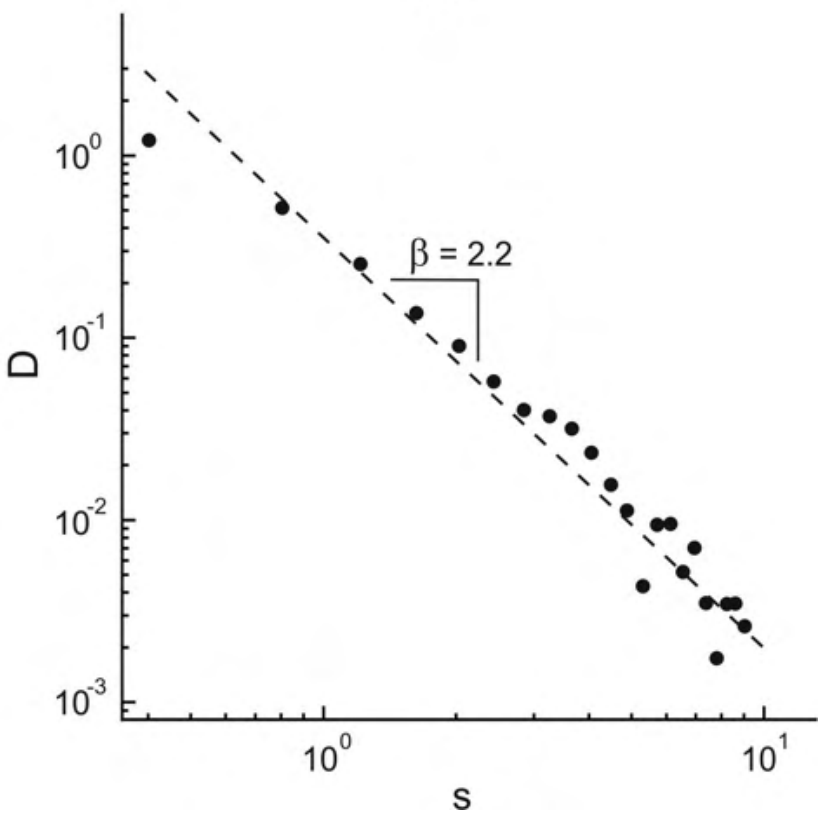

Figure 9

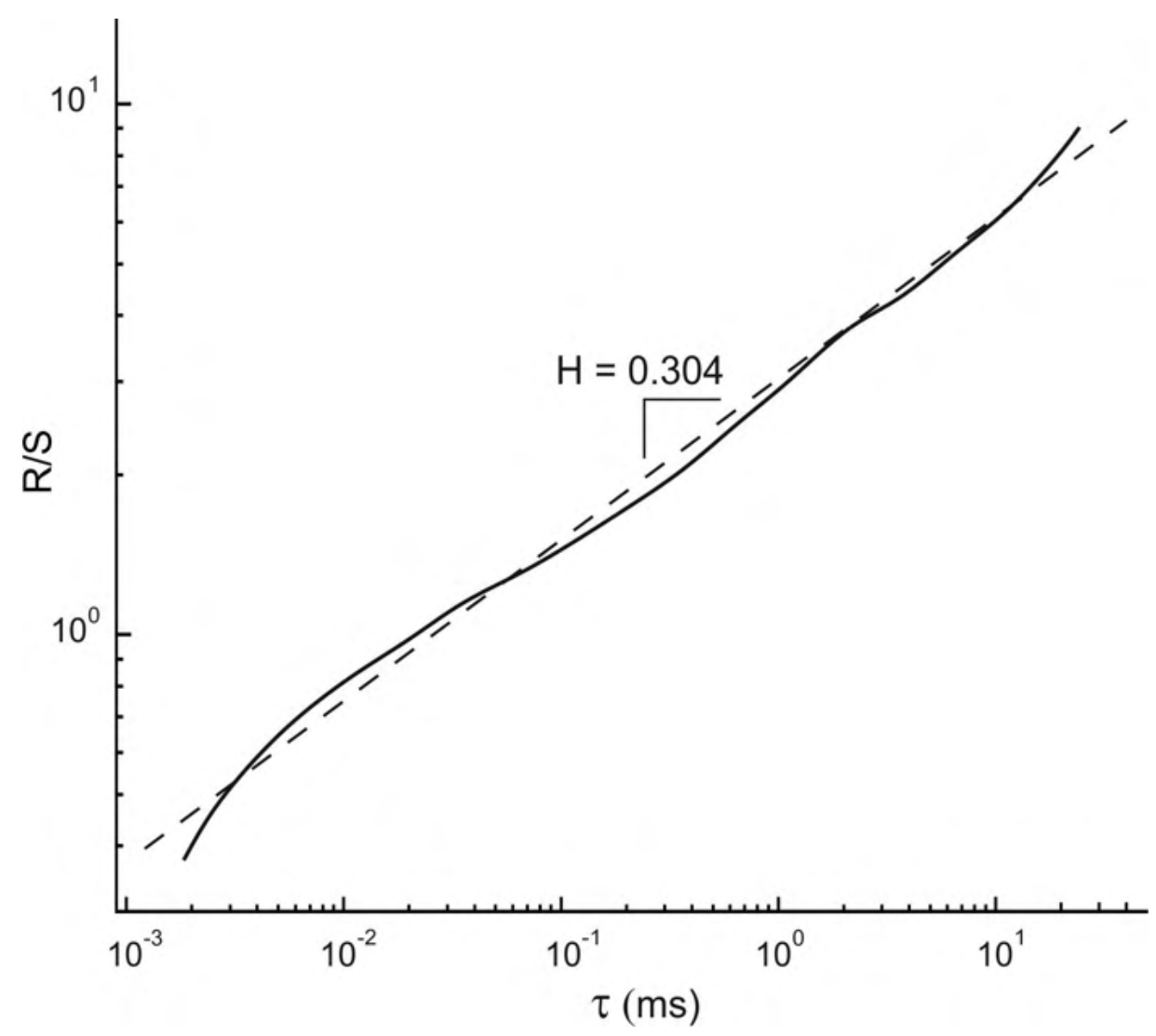

Figure 10 


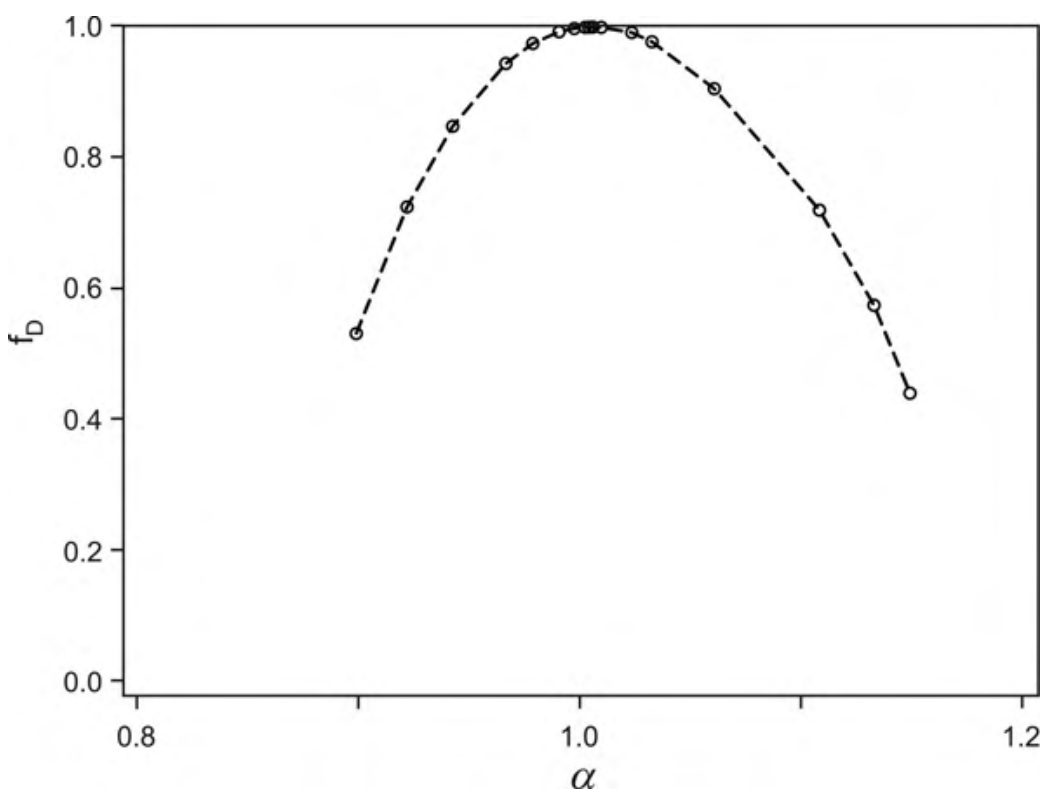

Figure 11

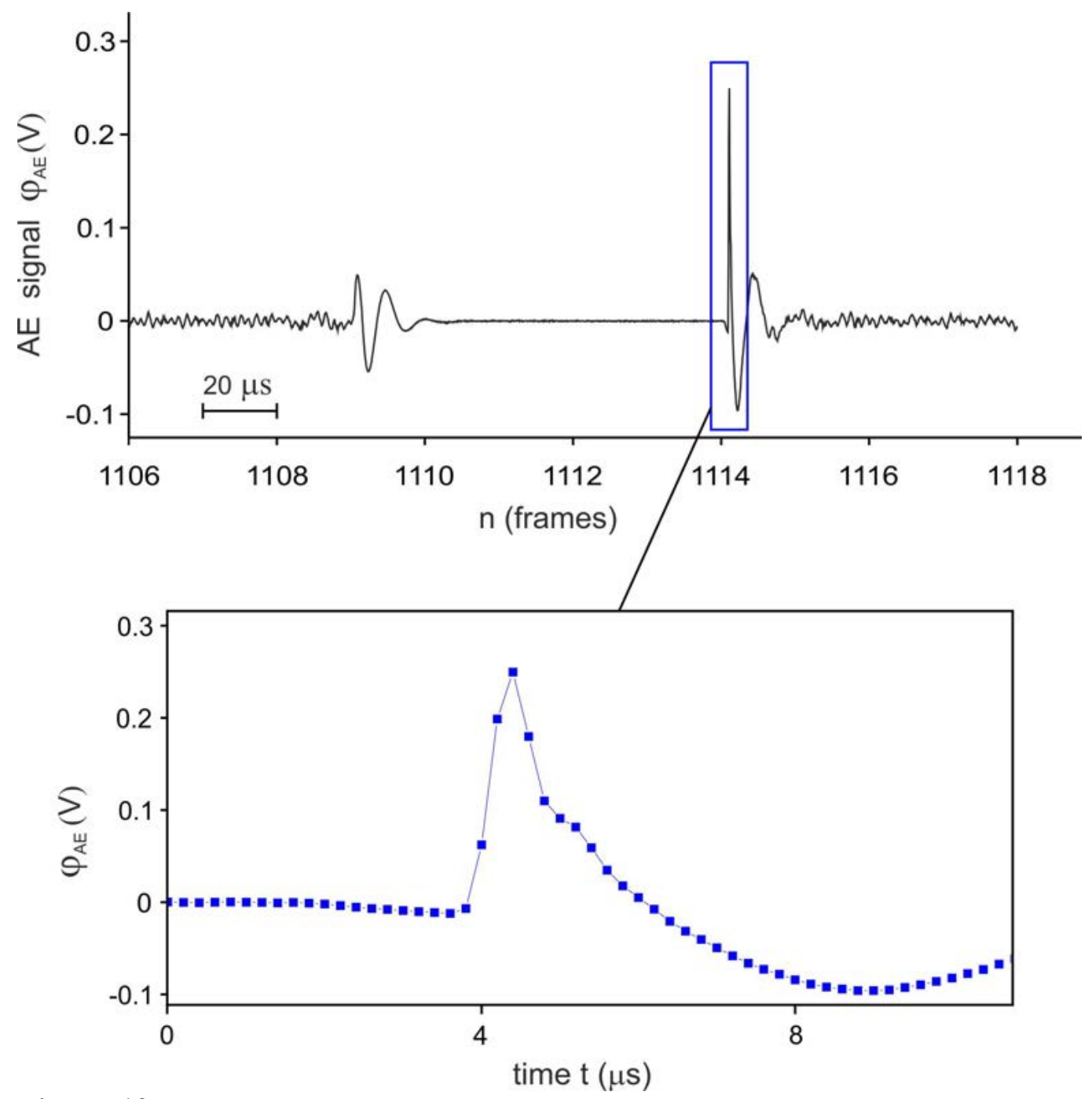

Figure 12 

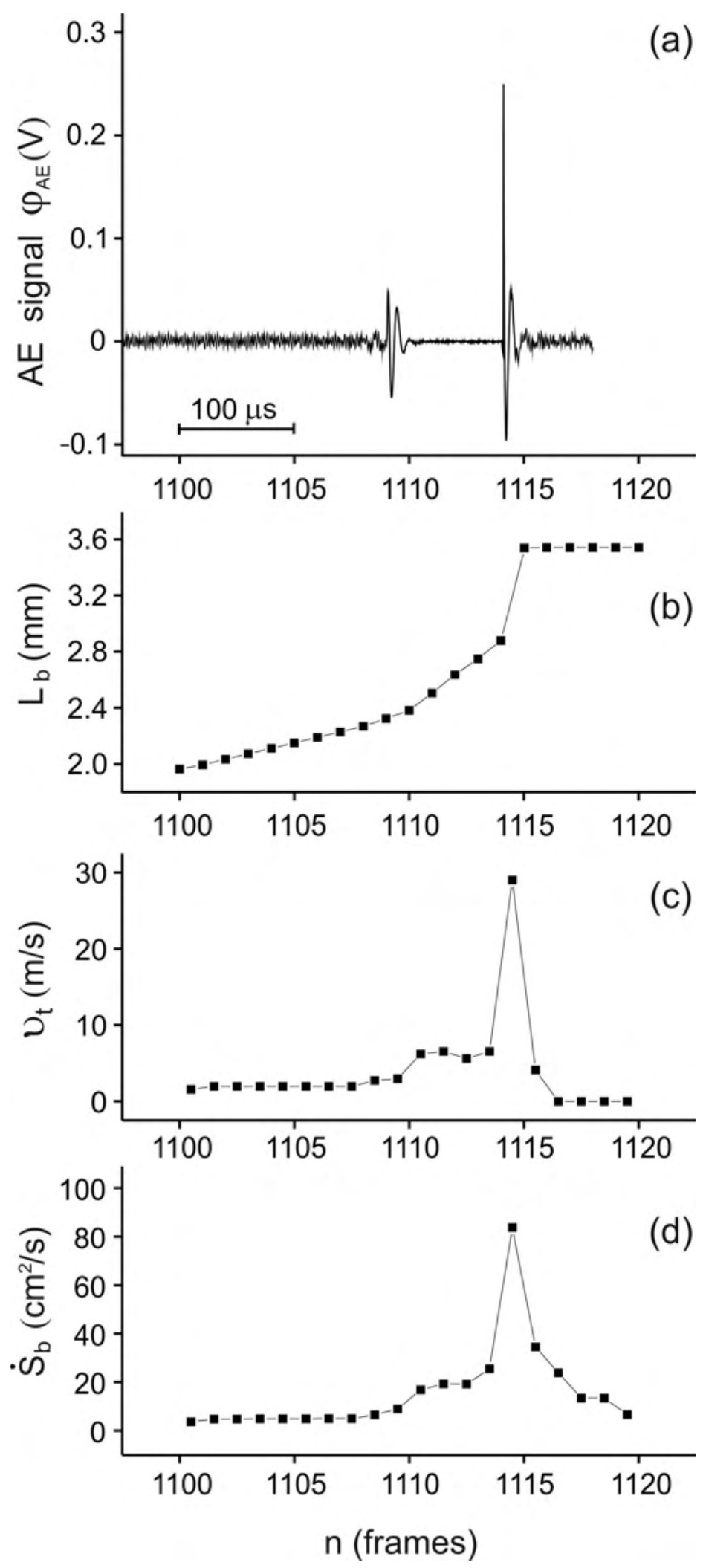

Figure 13 